\title{
Piezoelectric Passive Distributed Controllers for Beam Flexural Vibrations
}

\author{
U. ANDREAUS \\ F. DELL'ISOLA \\ Dipartimento di Ingegneria Strutturale e Geotecnica, La Sapienza, 00184 Roma, Italia
}

M. PORFIRI

Dottorato in Meccanica Teorica e Applicata, La Sapienza, 00184 Roma, Italia

Department of Engineering Science and Mechanics, Virginia Tech, Blacksburg, VA 24061, USA

(Received 8 November 2002; accepted 23 June 2003)

\begin{abstract}
Recent technological developments have made available efficient bender transducers based on the piezoelectric effect. In this paper an electrical circuit analog to the Timoshenko beam is synthesized using a Lagrangian method and by paralleling capacitive flux linkages to rotation and transverse displacement. A Piezo-ElectroMechanical (PEM) beam is conceived by uniformly distributing piezoelectric transducers on a beam and interconnecting their electric terminals via the found analog circuit, completed with suitable resistors. The high performance features of the synthesized novel circuit include the following. (i) The circuit topology is extremely reduced, the used components are all but one two - terminal elements, and the only two port network needed is an ideal transformer. (ii) One and the same dissipative circuit ensures a multiresonance coupling with the vibrating beam and the optimal electrical dissipation of mechanical vibrations energy. (iii) For a prototype of a PEM beam, the design of the analog circuit is possible and the obtained nominal values of the circuital elements ensure that can be technically realized without any external feeding. The insertion of resistors in the analog circuit is determined according to two optimality criteria (namely minimization of strain energy time envelope and maximization of vibration time rate decay), based on specific engineering needs. The former seems to be suitable for applications in fatigue phenomena and the latter when the amplitude of vibrations must be rapidly decreased, independently of the initial conditions.
\end{abstract}

Key Words: Smart structures, vibration control, piezoelectric transducers, analog circuits, distributed passive control

\section{INTRODUCTION}

\subsection{State of the Art}

The direct piezoelectric effect (electric charges produced on the surface of certain crystals by applied mechanical load) was experimentally discovered by the Curie brothers, Pierre and Jacques Curie, in 1880. One year later, in 1881, the converse piezoelectric effect (deformation of the crystals induced by an applied electric field) was deduced by Lippmann via thermodynamical considerations. The existence of the converse effect was immediately 
confirmed experimentally by the Curie brothers. Afterwards, the piezoelectric phenomenon was investigated by numerous scientists, among whom were Langevin, Voigt, and Mason (1950). Its technological applications were limited for a long period by the physical properties of piezoelectric ceramics. Indeed, these ceramics could not be produced in homogeneous sufficiently large specimens and were very brittle. Therefore, piezoelectric transducers could be used only in specific technological applications, e.g. to change mirror orientations in laser apparatuses, in vinyl disk recording devices, in radio-frequency electronic oscillators, etc. However, in the last two decades more performing transducers based on the piezoelectric effect have been designed and made available for technical and industrial applications, such as active and passive control of vibrations of flexible structures. (For more details on the novel concepts and architectures used in the design of piezoelectric transducers, see Niezreski et al., 2001.)

Among the first applications conceived for the developed family of transducers in the field of active vibration control there was the so-called "electronic damping" (see Forward, 1981; Forward and Swigert, 1981; Hanagud et al., 1985; Hanagud et al., 1992): a set of piezoceramic devices is placed on a host structure to sense and control the mechanical vibrations. The deformation of the sensing transducers results in electrical signals, which are conditioned by suitably designed feedback electronics and then applied to actuating transducers; the actuators convert the applied electrical energy into mechanical energy, transmitting mechanical control actions to the host structure.

Such a concept proved to be effective as the available actuators can exert forces of several hundreds of Newtons as a response of voltage signals of several hundreds of volts, without losing their dielectric properties or undergoing destructive strain deformations. Indeed, the most remarkable feature of the modern piezoelectric transducers is their capability of remaining in the linear range in the presence of strains of the order of $0.1 \%$ (see again Niezreski et al. (2001) or Crawley (1994)).

When active control systems are used, the piezoelectric transducers driving requires complex power amplifiers and associated precise sensing electronics, resulting in the consumption of a significant amount of electrical power.

Hagood and von Flotow (1991) investigate the possibility of damping mechanical vibrations by means of a piezoelectric transducer positioned on a structural element and shunted with completely passive electrical circuits. In particular, two different shunting circuits are considered: resistive (R) and resonant (LR). The method proposed in Hagood and von Flotow (1991) allows for an efficient single-mode control of structural vibrations, whenever the resonant circuit is tuned to the mechanical mode to be suppressed; nevertheless, the efficiency of the electromechanical coupling strongly depends on the position of the transducer over the host structure. Moreover, the technical feasibility of the passive piezoelectric controller proposed in Hagood and von Flotow (1991) is limited, since too large inductors are required to produce low-frequency electrical resonance with the small inherent capacitance of the piezoelectric transducer.

An extension of the shunting technique proposed in Hagood and von Flotow (1991) to multiple modes suppression has been made in Hollkamp (1994), where a single localized piezoelectric element is connected to a multifrequency electric resonator. The multimode damper considered by Hollkamp (1994) is not equally effective for the set of modes to be suppressed; furthermore, the used inductors are still characterized by huge inductances. 
The first attempts to exploit arrays of piezoelectric elements as transducers of an electrical distributed controller have been made by Kader et al. (2001) and dell'Isola and Vidoli (1998). Kader et al. (2001) propose an active controller exploiting piezoelectric elements either as sensors or as actuators, and design an optimal distributed electronic circuit interconnecting the sensors/actuators. The synthesized electronic circuit is active, hence an external power supply is needed to feed the interconnecting electronics. Dell'Isola and Vidoli (1998) propose to interconnect the piezoelectric transducers as the capacitors of a classical second-order transmission line constituted only by inductors and resistors. This passive electrical controller is effective only for the suppression of a single mechanical mode; hence the vibration suppression is not ensured outside a fixed narrow frequency range.

In Vidoli and dell' Isola (2000) it is shown that, in order to achieve an effective multimode control of mechanical vibrations, exploiting passive distributed interconnecting circuits, the spectrum of the differential operator governing the free vibrations of the electric controller should be as close as possible to the spectrum of the operator governing the mechanical vibrations.

Thus, Alessandroni et al. (2002) address the problem of finding an electrical interconnecting circuit governed by the Elastica equation (electric analog of the Euler beam). Two different networks are found: the first constituted by negative inductors, positive inductors and capacitors; the second by multiport transformers terminated by inductors and capacitors. The technical feasibility of the proposed networks is limited by the need to use electronic active circuits to simulate negative inductors, and the complexity of multiport transformers.

\subsection{Objectives}

In order to cure the drawbacks of the distributed controllers proposed in Alessandroni et al. (2002), we synthesize a lumped electrical circuit analog to a beam, constituted only by inductors, capacitors and elementary two-port transformers. To this aim we formulate in a Lagrangian form the beam evolution equations, and spatially discretize its Lagrangian functional.

A Piezo-ElectroMechanical (PEM) beam is designed, interconnecting an array of piezoelectric bender transducers bonded to a host beam via the found completely passive analog circuit. Assuming that a large number of transducers is positioned over the structural element, a continuous model for the PEM beam is adopted. Referring to an experimental setup, an aluminum PEM beam is designed using available lead zirconate titanate (PZT) transducers, showing the technical feasibility of the novel proposed device.

As a sample application, the analysis of a simply-supported PEM beam is performed via a standard modal analysis. Two optimality criteria for the damping of vibrations are proposed and proper dissipative networks are added to the analog circuit to accomplish optimality conditions. The achieved electrical dissipation will be shown to be independent of the beam vibration mode.

\section{SYNTHESIZING THE ANALOG CIRCUIT OF TIMOSHENKO BEAMS BY LAGRANGIAN METHODS}

In this section an analog circuit of the vibrating Timoshenko beam is synthesized. The method proposed exploits the basic version of the Euler finite difference method (employed 


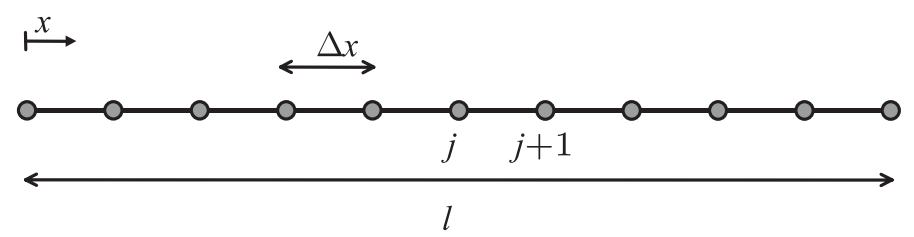

Figure 1. Discretization grid on the beam.

for instance in numerical analysis as an alternative to the finite element method; see, for example, Richards, 1977) and is articulated as follows.

1. We recall the variational principle governing the free vibrations of Timoshenko beams.

2. We define a mesh over the beam reference configuration and introduce (as a set of Lagrange coordinates) the sampled values of the fields describing the beam kinematics at the mesh nodes.

3. We approximate the infinite-dimensional Lagrangian for a Timoshenko beam via a finitedimensional Lagrangian in terms of introduced Lagrange coordinates.

4. With a well-known procedure (Gantmacher, 1980; Karplus and Soroka, 1959; Crandall et al., 1968) we synthesize a lumped electrical circuit, the evolution of which is governed by the so-found finite-dimensional Lagrangian.

Every material particle of the considered bending Timoshenko beam is labeled by an abscissa (varying in the interval $[0, l]$ where $l$ is the length) in its reference configuration and its state is characterized by a transverse displacement $u$ and a rotation of the beam crosssection $\vartheta$.

Let us subdivide the interval from 0 to $l$ on the $x$-axis putting equally spaced points $\Delta x=l / N$ units apart, where $N$ is the number of intervals (see Figure 1), and label

$$
x_{j}=j \Delta x
$$

for $j=0, \ldots, N$, so $x_{0}=0$ and $x_{N}=l$.

The Hamilton principle is given as

$$
\delta \int_{t_{0}}^{t_{1}} L \mathrm{~d} t=\delta \int_{t_{0}}^{t_{1}} K-E-W_{i n} \mathrm{~d} t=0
$$

where $L$ is the Lagrangian, $K$ is the total kinetic energy, $E$ is the total strain energy, $W_{i n}$ is the total input energy, and $t_{0}$ and $t_{1}$ are two arbitrary instants in time.

For a bending Timoshenko beam excited only at the ends, the Lagrangian reads (see, for instance, Soedel, 1993; Meirovitch, 2000)

$$
L=\int_{0}^{l} k(\dot{u}, \dot{\vartheta}) \mathrm{d} x-\int_{0}^{l} e\left(\vartheta, u^{\prime}, \vartheta^{\prime}\right) \mathrm{d} x-T_{0} u(0)-T_{l} u(l)-M_{0} \vartheta(0)-M_{l} \vartheta(l)
$$


where a superimposed dot denotes derivative with respect to time, and a prime indicates derivative with respect to space variable $x$. In equation (3), the density of kinetic energy $k$ and the density of elastic strain energy $e$ are given by

$$
\left\{\begin{array}{l}
k(\dot{u}, \dot{\vartheta})=\frac{1}{2}\left(\rho \dot{u}^{2}+I \dot{\vartheta}^{2}\right) \\
e\left(\vartheta, u^{\prime}, \vartheta^{\prime}\right)=\frac{1}{2}\left(K_{T}\left(u^{\prime}-\vartheta\right)^{2}+K_{M}\left(\boldsymbol{\vartheta}^{\prime}\right)^{2}\right)
\end{array} .\right.
$$

$T_{0}, T_{l}, M_{0}$, and $M_{l}$ represent the transversal loads and bending moments applied at the edges of the beam, $\rho$ is the mass density per unit length, $I$ is the cross-section moment of inertia, $K_{M}$ is the bending stiffness, and $K_{T}$ is the shear stiffness. Every constitutive parameter is assumed to be constant over the length of the beam.

The Euler-Lagrange equations associated with equations (3) and (4) are

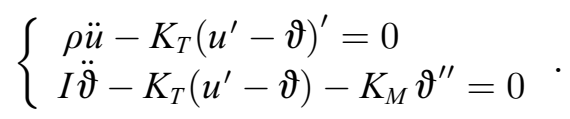

In order to approximate the infinite-dimensional system governed by the Lagrangian (3) with a finite-dimensional Lagrangian system, we consider the following extended numerical formula for the integration of the Lagrangian spatial density $k-e$ appearing in equation (3):

$$
L \simeq \Delta x \sum_{j=0}^{N} k_{j}\left(\dot{u}_{j}, \dot{\vartheta}_{j}\right)-\Delta x \sum_{j=0}^{N-1} e_{j}\left(\vartheta_{j}, u_{j}^{\prime}, \vartheta_{j}^{\prime}\right)-T_{0} u_{0}-T_{l} u_{N}-M_{0} \vartheta_{0}-M_{l} \vartheta_{N}
$$

Here, we have used the notation

$$
\left\{\begin{array}{l}
(\cdot)_{j}:=(\cdot)\left(x_{j}, t\right) \\
(\cdot)_{j}^{\prime}:=\frac{(\cdot)_{j+1}-(\cdot)_{j}}{\Delta x},
\end{array}\right.
$$

for the sampled values of the mechanical fields providing the values of the approximating step functions, as well as the Lagrange parameters describing the time evolution of the approximating finite-dimensional Lagrangian system. We explicitly remark that for estimating spatial derivatives a forward finite differences rule has been chosen.

We can express $k_{j}$ and $e_{j}$, i.e. the kinetic and strain energy densities at $x_{j}$, by

$$
\left\{\begin{array}{c}
e_{j} \simeq \frac{1}{2}\left(K_{T}\left(\frac{u_{j+1}-u_{j}}{\Delta x}-\vartheta_{j}\right)^{2}+K_{M}\left(\frac{\vartheta_{j+1}-\vartheta_{j}}{\Delta x}\right)^{2}\right) . \\
k_{j}=\frac{1}{2}\left(\rho \dot{u}_{j}^{2}+I \dot{\vartheta}_{j}^{2}\right)
\end{array}\right.
$$

Hence the Lagrangian of the Timoshenko beam can be conveniently approximated by 


$$
\begin{aligned}
L & \simeq \frac{1}{2} \Delta x \sum_{j=0}^{N}\left(\rho \dot{u}_{j}^{2}+I \dot{\vartheta}_{j}^{2}\right)+ \\
& -\frac{1}{2} \Delta x \sum_{j=0}^{N-1}\left(K_{T}\left(\frac{u_{j+1}-u_{j}}{\Delta x}-\vartheta_{j}\right)^{2}+K_{M}\left(\frac{\vartheta_{j+1}-\vartheta_{j}}{\Delta x}\right)^{2}\right)+ \\
& -T_{0} u_{0}-T_{l} u_{N}-M_{0} \vartheta_{0}-M_{l} \vartheta_{N}:=L^{f i n}
\end{aligned}
$$

The Lagrangian $L^{f i n}$ in equation (9) can be regarded as the Euler finite difference approximation for the Timoshenko beam Lagrangian given by equation (3).

The Euler-Lagrange equations obtained by equation (9) can be easily seen to govern the evolution of a completely passive lumped circuit, once an analogy between mechanical and electrical kinematical descriptors has been established. Therefore, we can directly obtain an electric analog for Timoshenko beams. The standard procedure for determining physical analogies requires the introduction of suitable scaling factors for the kinematical descriptors to be recognized as analogs. In the considered instance we introduce the scaling factors $\widetilde{u}$, $\widetilde{\vartheta}, \widetilde{\psi}, \widetilde{\varphi}$ respectively for transverse displacement, section rotation, and the two flux linkages, respectively.

Therefore, the problem of the synthesis of the analog circuit can be stated as follows.

Synthesis Problem. Find a $2 N+2$ degrees of freedom electrical circuit the Lagrangian of which is given by

$$
\begin{aligned}
L^{e l} & =\frac{1}{2} \Delta x \sum_{j=0}^{N}\left(\frac{\rho \widetilde{u}^{2}}{\widetilde{\psi}^{2}} \dot{\psi}_{j}^{2}+\frac{I \widetilde{\vartheta}^{2}}{\widetilde{\varphi}^{2}} \dot{\varphi}_{j}^{2}\right)+ \\
& -\frac{1}{2} \Delta x \sum_{j=0}^{N-1}\left(K_{T}\left(\frac{\widetilde{u}}{\widetilde{\psi}} \frac{\psi_{j+1}-\psi_{j}}{\Delta x}-\frac{\widetilde{\vartheta}}{\widetilde{\varphi}} \varphi_{j}\right)^{2}+K_{M}\left(\frac{\widetilde{\vartheta}}{\widetilde{\varphi}} \frac{\varphi_{j+1}-\varphi_{j}}{\Delta x}\right)^{2}\right)+ \\
& -T_{0} \frac{\widetilde{u}}{\widetilde{\psi}} \psi_{0}-T_{l} \frac{\widetilde{u}}{\widetilde{\psi}} \psi_{N}-M_{0} \frac{\widetilde{\vartheta}}{\widetilde{\varphi}} \varphi_{0}-M_{l} \frac{\widetilde{\vartheta}}{\widetilde{\varphi}} \varphi_{N}
\end{aligned}
$$

where $\psi_{j}$ and $\varphi_{j}$ represent the flux linkages of $2 N+2$ nodes measured with respect of a common reference ground.

Let us observe that to each mechanical sampling node must correspond two electrical nodes in the analog circuit, as shown in Figure 2: $\psi_{j}$ is the electrical analog of the mechanical deflection at node $j$, while $\varphi_{j}$ is the electrical analog of the rotation also at node $j$. The generalized variables of the electrical system are given by $\psi_{j}$ and $\varphi_{j}$ with $j$ varying between 0 and $N$. In this way it will be provided an analog circuit where the flux linkages represent the analog variables of the displacements and rotations, while the currents will be the electrical analogs of the bending moments and shear contact actions. ${ }^{1}$ 

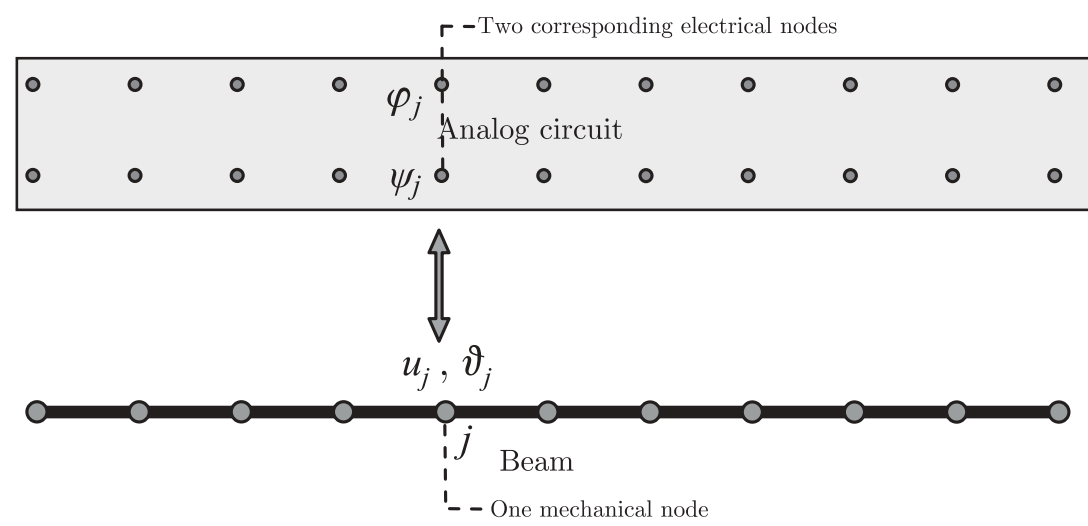

Figure 2. Schematic representation of the beam and its analog circuit.

Solution to the Synthesis Problem. The synthesis of the analog circuit is really straight forward, in fact let us connect each node of the circuit to a grounded capacitor, the value of which is

$$
C_{1}=\frac{\Delta x \rho \widetilde{u}^{2}}{\widetilde{\psi}^{2}}
$$

if the flux linkage at that node is analog to the beam deflection, or

$$
C_{2}=\frac{\Delta x I \widetilde{\vartheta}^{2}}{\widetilde{\varphi}^{2}},
$$

if the flux linkage at that node is analog to the beam rotation. Following the presented procedure we have designed a circuit the electric energy of which is given by the kinetic energy in equation (10): i.e. the mechanical kinetic energy finds its electrical analog in the electric (capacitive) energy. Interconnecting a pair of adjacent nodes the flux linkages of which are $\varphi_{j+1}$ and $\varphi_{j}$ via an inductor of inductance

$$
L_{1}=\frac{\Delta x \widetilde{\varphi}^{2}}{K_{M} \widetilde{\vartheta}^{2}},
$$

we immediately obtain the strain energy term (10), where the bending stiffness appears by means of magnetic (inductive) energy. Furthermore, in order to obtain the remaining term in the strain energy (10) - again by means of magnetic energy - a set of auxiliary nodes $\alpha_{j}$ must be introduced (see Figure 3), the flux linkages of which we want to fix equal to

$$
\alpha_{j}=\frac{\widetilde{\varphi} \widetilde{u}}{\widetilde{\vartheta} \widetilde{\psi}} \frac{\psi_{j+1}-\psi_{j}}{\Delta x} .
$$

This is done by means of an ideal transformer interconnected with a port between $\psi_{j+1}$ and $\psi_{j}$ and with the other port between ground and $\alpha_{j}$; the turns ratio is given by 


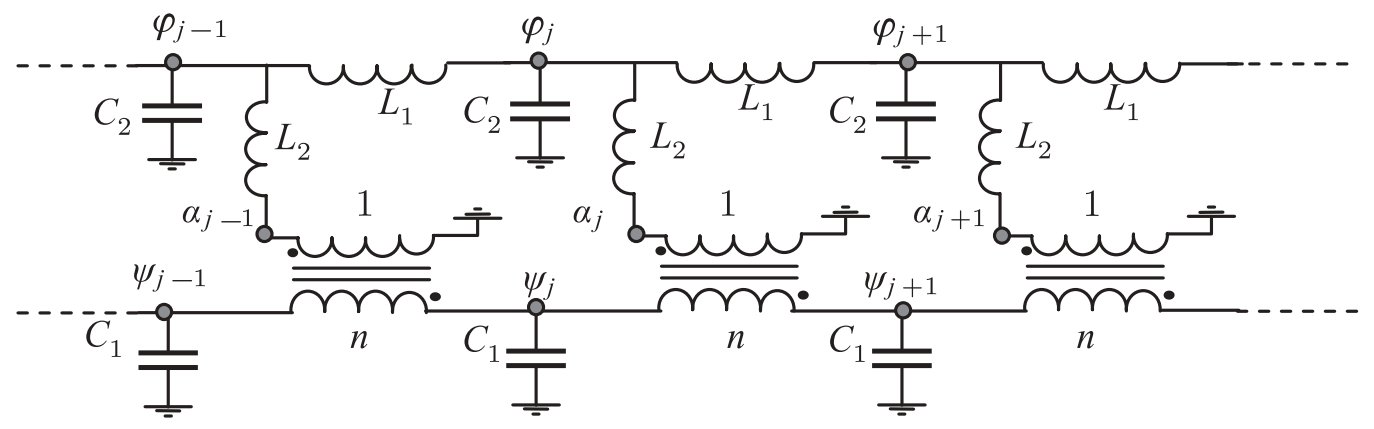

Figure 3. Internal modules of the electrical analog of the Timoshenko beam.

$$
n=\frac{\Delta x \widetilde{\vartheta} \widetilde{\psi}}{\widetilde{\varphi} \widetilde{u}}
$$

Finally the nodes $\alpha_{j}$ and $\varphi_{j}$ must be interconnected by an inductor of inductance

$$
L_{2}=\frac{\widetilde{\varphi}^{2}}{\Delta x K_{T} \widetilde{\vartheta}^{2}}
$$

The last four terms in equation (10), involving the input work of the concentrated external loads at the ends of the beam, have as electric analogs four current generators applied in parallel connections with the four capacitors at the boundary nodes of the circuit analog. For instance, the current generator at the node $\psi_{0}$ imposes a current of value

$$
I_{1}=T_{0} \frac{\widetilde{u}}{\widetilde{\psi}}
$$

the current imposed by the other three sources are similarly determined (see equation (12)).

In such a way we have designed a completely passive circuit the Lagrangian of which is given by

$$
\begin{aligned}
L^{e l} & =\frac{1}{2} \sum_{j=0}^{N}\left(C_{1} \dot{\psi}_{j}^{2}+C_{2} \dot{\varphi}_{j}^{2}\right)+ \\
& -\frac{1}{2} \sum_{j=0}^{N-1}\left\{\frac{1}{L_{2}}\left(\frac{\left(\psi_{j+1}-\psi_{j}\right)}{n}-\varphi_{j}\right)^{2}+\frac{1}{L_{1}}\left(\varphi_{j+1}-\varphi_{j}\right)^{2}\right\}+ \\
& -I_{1} \psi_{0}-I_{2} \psi_{N}-I_{3} \varphi_{0}-I_{4} \varphi_{N},
\end{aligned}
$$




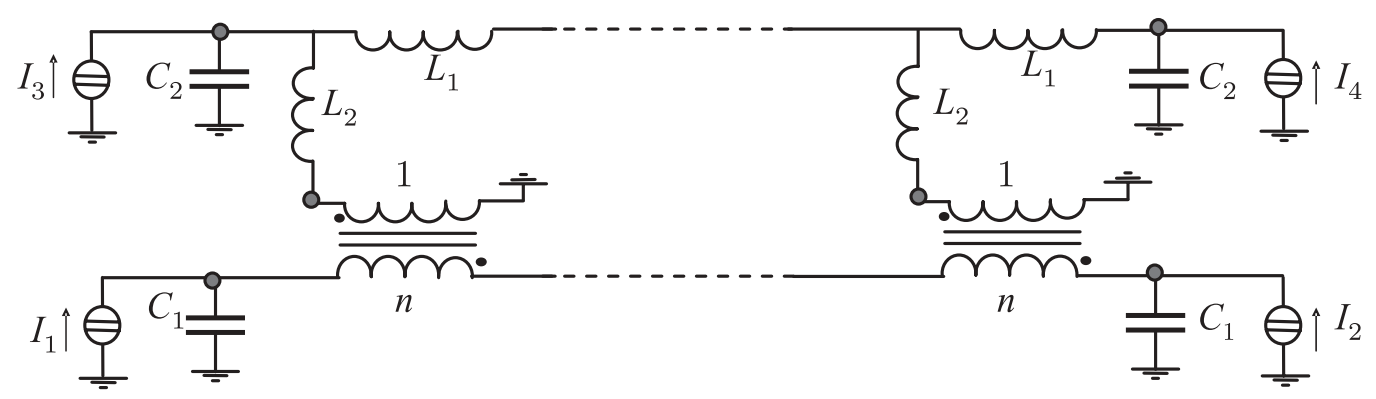

Figure 4. Boundary elements of the electrical analog.

with

$$
\left\{\begin{array} { l } 
{ C _ { 1 } = \Delta x \rho \widetilde { u } ^ { 2 } / \widetilde { \psi } ^ { 2 } } \\
{ C _ { 2 } = \Delta x I \widetilde { \vartheta } ^ { 2 } / \widetilde { \varphi } ^ { 2 } } \\
{ L _ { 1 } = \Delta x \widetilde { \varphi } ^ { 2 } / ( K _ { M } \widetilde { \vartheta } ^ { 2 } ) } \\
{ L _ { 2 } = \widetilde { \varphi } ^ { 2 } / ( \Delta x K _ { T } \widetilde { \vartheta } ^ { 2 } ) } \\
{ n = \frac { \Delta x \widetilde { \vartheta } \widetilde { \psi } } { \widetilde { \varphi } \widetilde { u } } }
\end{array} \quad \left\{\begin{array}{l}
I_{1}=T_{0} \frac{\widetilde{u}}{\widetilde{\psi}} \\
I_{2}=T_{l} \frac{\widetilde{\widetilde{u}}}{\widetilde{\psi}} \\
I_{3}=M_{0} \frac{\widetilde{\vartheta}}{\widetilde{\varphi}} \\
I_{4}=M_{l} \frac{\widetilde{\vartheta}}{\widetilde{\varphi}}
\end{array} .\right.\right.
$$

In Figure 3 the aforementioned analog circuit for the internal nodes of the Timoshenko beam is exhibited, while Figure 4 explains the connection of the current sources simulating the external loads at the edges.

Starting from relations (12) we immediately see that the following conditions independent of the adopted electromechanical scaling factors - hold:

$$
\left\{\begin{array}{l}
\frac{C_{1}}{C_{2}} n^{2}=\frac{\rho}{I}(\Delta x)^{2} \\
C_{2} L_{2}=\frac{I}{K_{T}} \\
C_{1} L_{1} n^{2}=\frac{\rho}{K_{M}}(\Delta x)^{4} \\
\frac{L_{1}}{L_{2}}=\frac{K_{T}}{K_{M}}(\Delta x)^{2}
\end{array}\right.
$$

These conditions imply that in the analog circuit of a given Timoshenko beam if two of the electrical elements among $C_{1}, L_{1}$, and $n$ have been chosen, then the other three are univocally determined by the geometry of the beam, its material properties and the step of the grid.

Before concluding the section we specify the approximation scheme in which the lumped circuit in Figure 3 verifies the Timoshenko equations. 


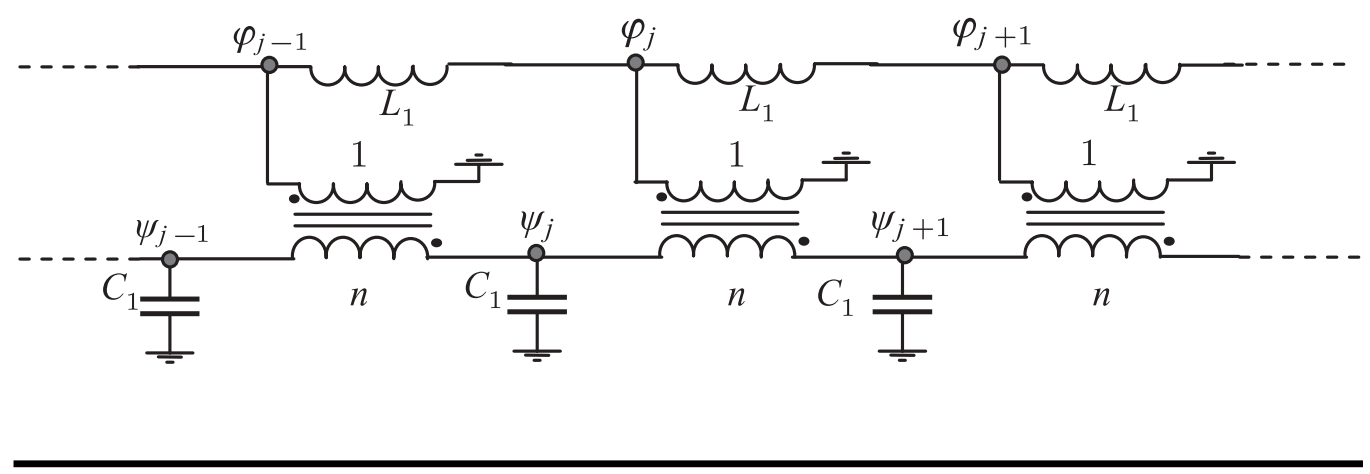

Figure 5. Electrical analog of the Euler beam.

Indeed, Euler-Lagrange equations for the internal nodes are

$$
\left\{\begin{array}{l}
C_{1} \ddot{\psi}_{j}-\frac{1}{L_{2} n^{2}}\left(\psi_{j+1}-2 \psi_{j}+\psi_{j-1}\right)+\frac{1}{L_{2} n}\left(\varphi_{j}-\varphi_{j-1}\right)=0 \\
C_{2} \ddot{\varphi}_{j}-\frac{1}{L_{2}}\left(\frac{\left(\psi_{j+1}-\psi_{j}\right)}{n}-\varphi_{j}\right)-\frac{1}{L_{1}}\left(\varphi_{j+1}-2 \varphi_{j}+\varphi_{j-1}\right)=0
\end{array}\right.
$$

and, as expected, the previous set of ordinary differential equations (ODEs) represents a finite difference approximation of the governing equations for the free vibrations of a Timoshenko beam given by equation (5). Analogous considerations hold for the transversality conditions.

Up to now the problems of designing electrical circuits to simulate external constraints acting on the beam have not been addressed. Nevertheless, the theory so far developed allows for an immediate solution of this problem.

As an example, consider a simply-supported beam, for which it is well known that the following boundary conditions hold:

$$
\left\{\begin{array}{l}
u(0, t)=0 \\
u(l, t)=0 \\
M(0, t)=0 \\
M(l, t)=0
\end{array}\right.
$$

Thus, the electrical analog of the simply-supported beam can be trivially synthesized short-circuiting the nodes $\psi_{0}$ and $\psi_{N}$ to ground and leaving open circuited $\varphi_{0}$ and $\varphi_{N}$, i.e. open-circuiting the two current generators $I_{3}$ and $I_{4}$.

In the following discussion we will focus our attention on a Euler beam, hence we will neglect the shear deformability by assuming $K_{T} \rightarrow \infty$ and we will disregard the rotatory inertia assuming $I=0$. These assumptions are motivated by the idea to control only lowfrequency modes of structures; hence, in this framework the elastica equations are quite satisfactory.

The electric analog of the Euler beam can be thus immediately designed from the analog of the Timoshenko beam by short-circuiting the inductor $L_{2}$ and opening the capacitor $C_{2}$, as depicted in Figure 5. 


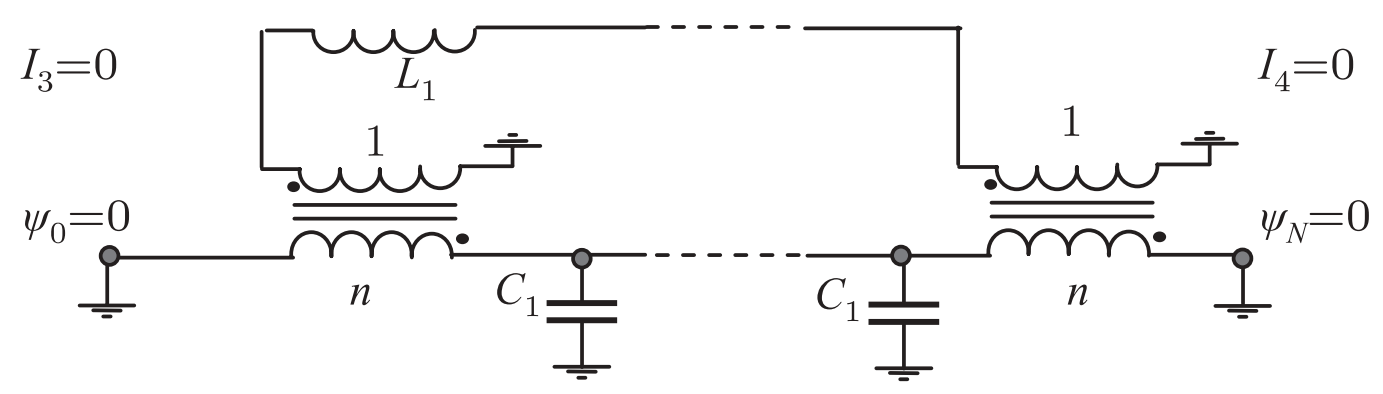

Figure 6. Boundary elements of the circuit analog to a simply-supported Euler beam.

The Lagrangian for the Euler beam circuit analog becomes

$$
\begin{aligned}
L^{e l} & =\frac{1}{2} \sum_{j=0}^{N} C_{1} \dot{\psi}_{j}^{2}-\frac{1}{2} \sum_{j=1}^{N-1} \frac{1}{L_{1} n^{2}}\left(\psi_{j+1}-2 \psi_{j}+\psi_{j-1}\right)^{2}+ \\
& -\frac{1}{2} \frac{1}{L_{1}}\left(\frac{\psi_{N}-\psi_{N-1}}{n}-\varphi_{N}\right)^{2}-I_{1} \psi_{0}-I_{2} \psi_{N}-I_{3} \frac{\psi_{1}-\psi_{0}}{n}-I_{4} \varphi_{N} .
\end{aligned}
$$

The Euler-Lagrange equations for the internal nodes become

$$
C_{1} \ddot{\psi}_{j}+\frac{1}{L_{1} n^{2}}\left(\psi_{j+2}-4 \psi_{j+1}+6 \psi_{j}-4 \psi_{j-1}+\psi_{j-2}\right)=0, \quad j=2, \ldots, N-2,
$$

which is immediately recognized as a central finite difference approximation of the elastica.

Let us now concentrate again on the electrical analog of the simply-supported beam, for which the boundary elements of the analog circuit are shown in Figure 6.

The governing equations are

$$
\left\{\begin{array}{l}
C_{1} \ddot{\psi}_{j}+\frac{1}{L_{1} n^{2}}\left(\psi_{j+2}-4 \psi_{j+1}+6 \psi_{j}-4 \psi_{j-1}+\psi_{j-2}\right)=0, \quad j=2, \ldots, N-2 \\
C_{1} \ddot{\psi}_{1}+\frac{1}{L_{1} n^{2}}\left(5 \psi_{1}-4 \psi_{2}+\psi_{3}\right)=0 \\
C_{1} \ddot{\psi}_{N-1}+\frac{1}{L_{1} n^{2}}\left(5 \psi_{N-1}-4 \psi_{N-2}+\psi_{N-3}\right)=0 \\
\psi_{0}=\psi_{N}=0
\end{array}\right.
$$




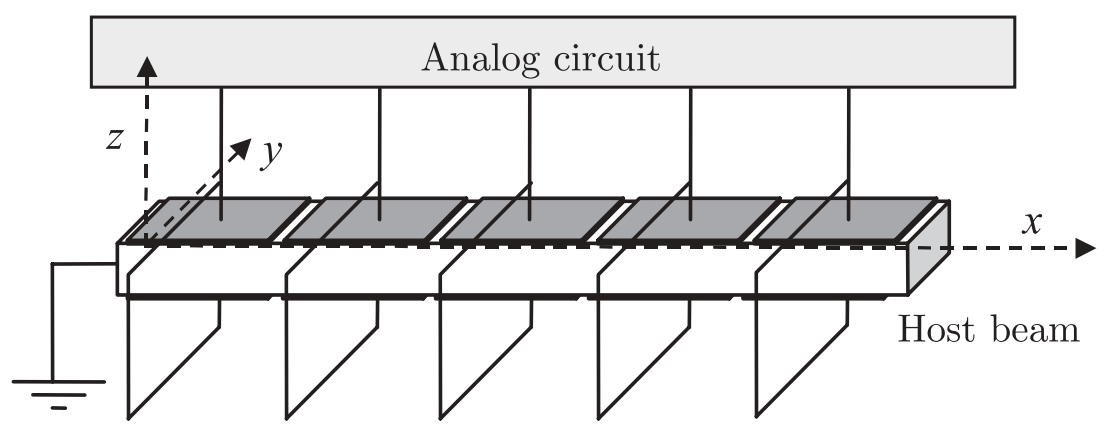

Figure 7. PEM beam.

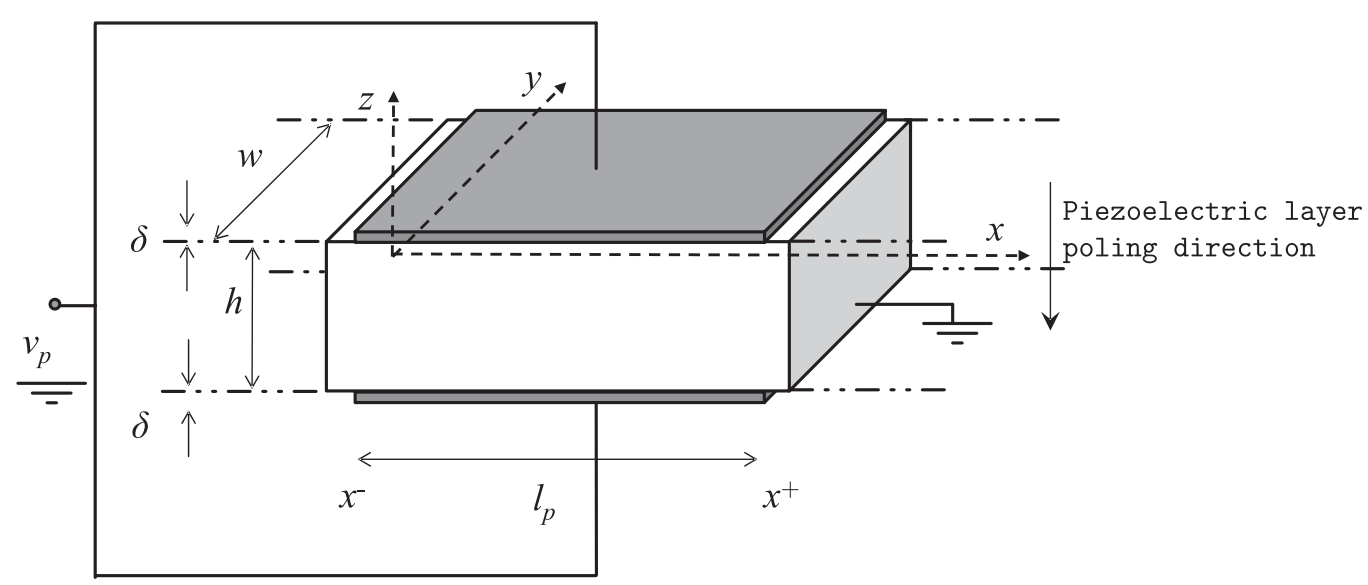

Figure 8. Schematic representation of a PEM element.

\section{PEM BEAM}

In this section we define a novel smart structure which we have called (dell'Isola and Vidoli, 1998; Alessandroni et al., 2002) a PEM beam, making use of the analog circuit designed in the previous section. Such a circuit will interconnect (see Figure 7) an array of piezoelectric transducers uniformly distributed over the structural element ${ }^{2}$, which in this paper will be assumed to be a rectangular cross-section beam.

The array of piezoelectric transducers will be positioned on the upper and lower surface of the beam, each pair of patches will be in the so-called "bender" configuration, i.e. in parallel connection, with a terminal connected to the ground and with the same poling directions (see Figure 8). The ungrounded piezoelectric terminals will be interconnected via the analog circuit of the Euler beam synthesized in the previous section where the patches will replace the capacitors in the analog circuit. 


\subsection{Constitutive Equations for a Bender Transducer}

Our analysis starts by introducing an elementary model for a bender piezoelectric transducer employed in PEM beams. In Figure 8 a pair of piezoelectric elements in the bender configuration is shown surface-bounded to the beam to be controlled.

Let us assume the thickness $\delta$ of the piezoelectric transducers to be negligible compared to the thickness of the beam $h$ and that a perfect bonding between the beam and the actuators is realized.

Moreover, we will assume that in each patch the stress and the electric field are of the following forms (see Figure 8):

$$
[T]=\left[\begin{array}{ccc}
\sigma_{x} & 0 & \tau_{x z} \\
0 & 0 & 0 \\
\tau_{x z} & 0 & 0
\end{array}\right] ; \quad \frac{\partial \sigma_{x}}{\partial y}=\frac{\partial \sigma_{x}}{\partial z}=0, \frac{\partial \tau_{x z}}{\partial y}=0 .
$$

and

$$
[E]=\left[\begin{array}{c}
0 \\
0 \\
E_{z}
\end{array}\right] ; \quad \frac{\partial E_{z}}{\partial x}=\frac{\partial E_{z}}{\partial y}=\frac{\partial E_{z}}{\partial z}=0 .
$$

For more details, see Hanagud et al. (1992), Hagood and von Flotow (1991) and Crawley and de Luis (1987).

These assumptions are physically well-grounded when $\delta<<h$ (see IEEE, 1987).

Once the transversely isotropic constitutive equations for piezoelectric three-dimensional (3D) continua are assumed (see, for example, IEEE, 1987; Mason, 1950), the onedimensional constitutive equations for the piezoelectric patches are given by

$$
\left[\begin{array}{c}
\varepsilon_{x} \\
D_{z}
\end{array}\right]=\left[\begin{array}{cc}
s_{11}^{E} & -d_{31} \\
-d_{31} & \epsilon_{3}^{T}
\end{array}\right]\left[\begin{array}{l}
\sigma_{x} \\
E_{z}
\end{array}\right]
$$

where $\varepsilon_{x}$ is the strain, $D_{z}$ is the electrical displacement, $d_{31}$ is the piezoelectric constant, $s_{11}^{E}$ is the elastic compliance at constant electric field, and $\epsilon_{3}^{T}$ is the dielectric constant at constant stress.

We assume that the variation of the strain along the thickness of the actuators is negligible, so that we can assume

$$
\left\{\begin{aligned}
\varepsilon_{x}^{u} & =-\frac{h}{2} u(x, t)^{\prime \prime} \\
\varepsilon_{x}^{l} & =+\frac{h}{2} u(x, t)^{\prime \prime}
\end{aligned}\right.
$$

where the superscripts $u$ and $l$ indicate, respectively, the upper or lower patch.

Furthermore, we have assumed also that the electrical displacement field is constant over the thickness of the patch (see equation (20)). As the flux of $D_{z}$ over the two-patch electrodes is equal to the charge stored in each actuator, then integrating the electric displacement field over the surface of the piezo-layer, it is easy to obtain 


$$
Q_{p}(t)=2 \frac{w l_{p}\left(s_{11}^{E} \epsilon_{3}^{T}-d_{31}^{2}\right)}{s_{11}^{E} \delta} v_{p}(t)-\frac{h w d_{31}}{s_{11}^{E}}\left(u^{\prime}\left(x^{+}\right)-u^{\prime}\left(x^{-}\right)\right),
$$

where $Q_{p}$ is the charge stored in the bender transducer, $w$ and $l_{p}$ are respectively the width and length of the actuator, $u^{\prime}\left(x^{ \pm}\right)$are the rotations of the cross-sections of the beam at the edges of the patch and $v_{p}$ is the voltage drop measured with respect to the ground. Sometimes the electromechanical coupling coefficient $k_{31}$ is used and the previous expression takes the form

$$
Q_{p}(t)=2 \frac{w l_{p} \epsilon_{3}^{T}}{\delta}\left(1-k_{31}^{2}\right) v_{p}(t)-\frac{h w d_{31}}{s_{11}^{E}}\left(u^{\prime}\left(x^{+}\right)-u^{\prime}\left(x^{-}\right)\right)
$$

with

$$
k_{31}=d_{31} \sqrt{\frac{1}{s_{11}^{E} \epsilon_{3}^{T}}} .
$$

The bending moment at a generic transversal section between $x^{+}$and $x^{-}$is equal to

$$
M(x, t)=-w \int_{-h / 2-\delta}^{h / 2+\delta} \sigma_{x} z \mathrm{~d} z .
$$

Considering the constitutive relation in equation (21) and neglecting higher-order terms we obtain

$$
M(x, t)=\left(E I+\frac{h^{2} w \delta}{2 s_{11}^{E}}\right) u^{\prime \prime}(x, t)+\frac{h w d_{31}}{s_{11}^{E}} v_{p}(t),
$$

where $I$ is the moment of inertia of the cross-section and $E$ is the Young modulus of the beam.

Hence the constitutive equations for the PEM beam element in Figure 8 can be written as

$$
\left\{\begin{array}{l}
M(x, t)=K_{M} u^{\prime \prime}(x, t)+k_{m e} v_{p}(t) \\
Q_{p}(t)=k_{e e} v_{p}(t)-k_{m e}\left(u^{\prime}\left(x^{+}\right)-u^{\prime}\left(x^{-}\right)\right)
\end{array},\right.
$$

with

$$
\left\{\begin{array}{l}
K_{M}=E I+\frac{h^{2} w \delta}{2 s_{11}^{E}} \\
k_{e e}=2 \frac{w\left(s_{11}^{E} \epsilon_{3}^{T}-d_{31}^{2}\right)}{s_{11}^{E} \delta} l_{p}=2 \frac{w l_{p} \epsilon_{3}^{T}}{\delta}\left(1-k_{31}^{2}\right) \\
k_{m e}=\frac{h w d_{31}}{s_{11}^{E}}
\end{array}\right.
$$

Assuming that the number of patches positioned over the beam (each of them of a length equal to $\Delta x$, i.e. $l_{p}=\Delta x$ ) is large enough, it is conceivable to assume the voltage drop and the stored charge to be distributed variables over the length of the beam. Thus the constitutive equations become 


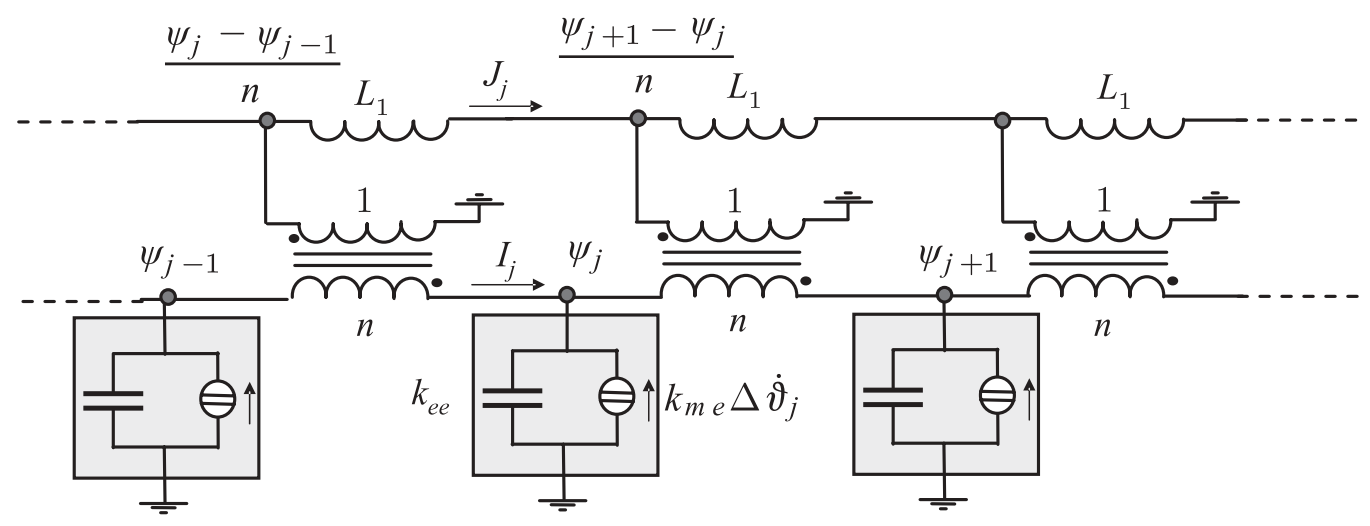

Figure 9. Electric analog connected to the bender transducers. $\left(\Delta \dot{\vartheta}_{j}:=\dot{u}_{j+1}^{\prime}-\dot{u}_{j}^{\prime}\right)$.

$$
\left\{\begin{array}{l}
M(x, t)=K_{M} u^{\prime \prime}(x, t)+k_{m e} \dot{\psi}(x, t) \\
q(x, t)=\frac{k_{e e}}{\Delta x} \dot{\psi}(x, t)-k_{m e} u^{\prime \prime}(x, t)
\end{array},\right.
$$

where $q$ is the charge per unit length of the PEM beam and $\psi$ is the flux linkage.

Equation (30) follows from the homogenized model of PEM beams, which assumes that the piezo-layer introduces:

1. a distributed capacitance and charge source proportional to the curvature of the beam (electrical viewpoint);

2. an additional bending stiffness and a surplus of bending moment driven by the piezoelectric voltage drop (mechanical viewpoint).

\subsection{Non-Dissipative PEM Beam}

\subsubsection{Deduction of Evolution Equations}

Interconnecting the bender transducers as shown in Figure 9 (i.e. interconnecting the bender transducer between $x_{j}$ and $x_{j+1}$ as the capacitor at node $\psi_{j}$ of the electrical analog circuit in Figure 5) the analysis of the exhibited circuit is easily performed. Indeed, the balance of currents at node $\psi_{j}$ and equation $(28)_{2}$ specifying how the current sources are controlled by mechanical time rate strains implies

$$
k_{e e} \ddot{\psi}_{j}-k_{m e}\left(\dot{u}^{\prime}\left(x_{j+1}\right)-\dot{u}^{\prime}\left(x_{j}\right)\right)=I_{j}-I_{j+1} .
$$

On the other hand, because of the constitutive equations of ideal transformers and inductors we obtain (see Figures 5 and 9): 


$$
\left\{\begin{array}{l}
I_{j}=\frac{1}{n}\left(J_{j-1}-J_{j}\right) \\
\varphi_{j-1}=\frac{1}{n}\left(\psi_{j}-\psi_{j-1}\right) \\
J_{j-1}=\frac{1}{L_{1}}\left(\frac{1}{n}\left(\psi_{j}-\psi_{j-1}\right)-\frac{1}{n}\left(\psi_{j-1}-\psi_{j-2}\right)\right)
\end{array} .\right.
$$

Substituting equation (32) into equation (31) leads to the following evolution equations for the "mechanically fed" analog circuit:

$$
\begin{aligned}
k_{e e} \ddot{\psi}_{j}+\frac{1}{L_{1} n^{2}}\left(\psi_{j+2}-4 \psi_{j+1}+6 \psi_{j}-4 \psi_{j-1}+\psi_{j-2}\right) & =k_{m e}\left(\dot{u}^{\prime}\left(x_{j+1}\right)-\dot{u}^{\prime}\left(x_{j}\right)\right), \\
j & =2, \ldots, N-2 .
\end{aligned}
$$

When the homogenized model can be used equation (33) becomes

$$
\frac{(\Delta x)^{3}}{L_{1} n^{2}} \psi^{\mathrm{IV}}+\frac{k_{e e}}{(\Delta x)} \ddot{\psi}-k_{m e} \dot{u}^{\prime \prime}=0 .
$$

Considering the constitutive equations (30), the equation for the free vibrations of the beam in terms of the deflection (see, for example, Soedel, 1993) becomes

$$
K_{M} u^{\mathrm{IV}}+\rho \ddot{u}+k_{m e} \dot{\psi}^{\prime \prime}=0,
$$

where $\rho$ is the density of mass per unit length of the PEM beam, i.e.

$$
\rho=2 \rho_{p} \delta w+\rho_{b} w h .
$$

$\rho_{p}$ is the density of mass per unit volume of the patches and $\rho_{b}$ is the density per unit volume of the material constituting the beam.

Summarizing, the homogenized governing equations of the PEM beam are

$$
\left\{\begin{array}{l}
K_{M} u^{\mathrm{IV}}+\rho \ddot{u}+k_{m e} \dot{\psi}^{\prime \prime}=0 \\
\frac{1}{\lambda v^{2}} \psi^{\mathrm{IV}}+\gamma \ddot{\psi}-k_{m e} \dot{u}^{\prime \prime}=0
\end{array}, \quad\left\{\begin{array}{l}
\gamma=\frac{k_{e e}}{(\Delta x)}=2 \frac{w \epsilon_{3}^{T}}{\delta}\left(1-k_{31}^{2}\right) \\
v=\frac{n}{(\Delta x)} \\
\lambda=\frac{L_{1}}{(\Delta x)}
\end{array} ;\right.\right.
$$

where $\gamma, v$, and $\lambda$ are respectively the piezo-capacitance per unit length, turns-ratio per unit length and inductance per unit length.

In order to guarantee an efficient multimodal control based on internal resonance, it is necessary, as proved in Vidoli and dell'Isola (2000), to tune the electrical circuit so as to 
Table 1. Properties and dimensions of the host beam.

\begin{tabular}{ccc}
\hline Coefficient & Value & Units \\
\hline$l$ & $510 \times 10^{-3}$ & $\mathrm{~m}$ \\
$w$ & $40 \times 10^{-3}$ & $\mathrm{~m}$ \\
$h$ & $4 \times 10^{-3}$ & $\mathrm{~m}$ \\
$E$ & $70 \times 10^{9}$ & $\mathrm{~Pa}$ \\
$\rho_{b}$ & 2700 & $\mathrm{~kg} \mathrm{~m}^{-3}$ \\
\hline
\end{tabular}

$$
\gamma v^{2} \lambda=\frac{\rho}{K_{M}} .
$$

Furthermore, it is very useful to non-dimensionalize the previous coupled equations in order to estimate the efficiency of the gyroscopic coupling (for some details on gyroscopic systems, see for example, Meirovitch, 1974, 1975; Yang, 1991) ensured by the coefficient $k_{m e}$. Thus, introducing as usual the scaling parameters $\widetilde{u}, \widetilde{\psi}, l$, and $\varpi$ for the deflection, the flux linkage, length and frequency ${ }^{3}$, the dimensionless coupled equations become

$$
\left\{\begin{array}{l}
u^{\mathrm{IV}}+\alpha^{4} \ddot{u}+\beta_{m e}^{2} \dot{\psi}^{\prime \prime}=0 \\
\psi^{\mathrm{IV}}+\alpha^{4} \ddot{\psi}-\beta_{e m}^{2} \dot{u}^{\prime \prime}=0
\end{array}, \quad\left\{\begin{array}{l}
\alpha^{4}=\frac{\varpi^{2} \rho l^{4}}{K_{M}}=\varpi^{2} \gamma v^{2} \lambda l^{4} \\
\beta_{m e}^{2}=\frac{\varpi l^{2} k_{m e}}{K_{M}} \frac{\widetilde{\psi}}{\widetilde{u}} \\
\beta_{e m}^{2}=\varpi l^{2} k_{m e} \lambda v^{2} \frac{\widetilde{u}}{\widetilde{\psi}}
\end{array} ;\right.\right.
$$

where with an abuse of notation the dimensional and dimensionless variables are denoted with the same symbols.

In order to maintain the form of the gyroscopic coupling, it is sufficient to choose the scaling parameters for the flux linkage and for the deflection as follows ${ }^{4}$ :

$$
\frac{\widetilde{\psi}}{\widetilde{u}}=\sqrt{\frac{\rho}{\gamma}}
$$

so we obtain:

$$
\beta_{m e}^{2} \equiv \beta_{e m}^{2}=\frac{\varpi l^{2} k_{m e}}{K_{M}} \sqrt{\frac{\rho}{\gamma}}=: \beta^{2}
$$

\subsubsection{Design of a Prototype of a PEM Beam}

In order to assess the physical realizability and the efficiency of the proposed device, let us consider an aluminum beam, the geometry of which is presented in the Table 1.

Let us position nine bender transducers constituted by piezoceramic patches (T110-H4E602 (PSI-5H4); see Piezo Systems, Inc., Cambridge, MA, http://www.piezo.com) made of PZT. (For details on the different types of piezoelectric actuation, see Niezreski et al., 2001).

The characteristics of these piezoceramic transducers are presented in Table 2. 
Table 2. Properties and dimensions of the T110-H4E-602 (PSI-5H4).

\begin{tabular}{ccc}
\hline Coefficient & Value & Units \\
\hline$\rho_{p}$ & 7800 & $\mathrm{~kg} \mathrm{~m}^{-3}$ \\
$\left(s_{11}^{E}\right)^{-1}$ & $62 \times 10^{9}$ & $\mathrm{~Pa}$ \\
$d_{31}$ & $-320 \times 10^{-12}$ & $\mathrm{~m} \mathrm{~V}^{-1}$ \\
$\epsilon_{3}^{T}$ & $3800 \times 8.85 \times 10^{-12}$ & $\mathrm{~F} \mathrm{~m}^{-1}$ \\
$\delta$ & $0.267 \times 10^{-3}$ & $\mathrm{~m}$ \\
\hline
\end{tabular}

The step for the finite difference grid is given by

$$
\Delta x=l / 9 \simeq 56.667 \times 10^{-3} \mathrm{~m} .
$$

The stiffness and mass per unit length of the PEM beam can be evaluated to be

$$
\left\{\begin{array}{l}
K_{M}=E I+\frac{h^{2} w \delta}{2 s_{11}^{E}} \simeq 20.23 \mathrm{Nm}^{2} \\
\rho=2 \rho_{p} \delta w+\rho_{b} w h \simeq .5986 \mathrm{~kg} \mathrm{~m}^{-1}
\end{array},\right.
$$

while the coupling parameter $k_{m e}$ is given by

$$
k_{m e}=\frac{h w d_{31}}{s_{11}^{E}} \simeq-3.174 \times 10^{-3} \mathrm{Nm} \mathrm{V}^{-1} .
$$

The product of inductance per unit length and the square of the turns ratio per unit length is given by

$$
v^{2} \lambda=\frac{\rho}{2 \frac{w \epsilon_{3}^{T}}{\delta}\left(1-k_{31}^{2}\right) K_{M}} \simeq 3620 \mathrm{H} \mathrm{m}^{-3} .
$$

Using transformers the turns ratio of which is equal to 10 the inductances to be used should be

$$
L_{1} \simeq 6.587 \mathrm{mH}
$$

Let us explicitly remark that the extremely reduced values of the used inductances and of the transformer turns ratio lead to a completely passive realization of the whole electrical analog network.

In the following we will choose as the angular frequency scaling parameter, the angular frequency of the first mode of the simply-supported PEM beam with all the bender actuators short-circuited:

$$
\varpi=\frac{\pi^{2}}{l^{2}} \sqrt{\frac{K_{M}}{\rho}}=220.6 \mathrm{rad} \mathrm{s}^{-1} .
$$




\subsection{Dissipative PEM beam}

Alessandroni et al. (2002) have studied the vibration damping induced by a set of resistors in parallel connection with the PZT actuators. In this paper we want to consider a different type of electric dissipation. In particular, we will show that connecting the resistors between the PZT capacitors, i.e. connecting the nodes $\psi_{j+1}$ and $\psi_{j}$ also by a resistor, it is possible to obtain the same damping factor for the evolution of each mode. Furthermore we will approach the optimization problem defining a criterion which seems to be closer to practical engineering problems.

Let us introduce in the coupled set of dimensionless homogenized evolution equations an electrical dissipation of the following differential form

$$
D[\dot{\psi}]=\sum_{k=0}^{M} y_{2 k} \frac{\partial^{2 k} \dot{\psi}}{\partial x^{2 k}},
$$

where the constant $M$ is a prescribed number and $y_{2 k}$ are real constants ${ }^{5}$. Thus, the governing equations for the dissipative PEM beam are

$$
\left\{\begin{array}{l}
u^{\mathrm{IV}}+\alpha^{4} \ddot{u}+\beta^{2} \dot{\psi}^{\prime \prime}=0 \\
\psi^{\mathrm{IV}}+\alpha^{4} \ddot{\psi}-\beta^{2} \dot{u}^{\prime \prime}-D[\dot{\psi}]=0
\end{array} .\right.
$$

The class of dissipation operators (48) has been chosen for the following reasons.

1. As shown in Figure 10, it is easy to synthesize a circuit governed by equation $(49)_{2}$ with $\beta=0$.

2. It is of Rayleigh type.

3. In the case of a simply-supported PEM beam it represents a proportional damping.

4. As will be shown in Section 4, in it one can find a dissipation operator which is optimal simultaneously for all vibration modes.

In the following section we will find, according to two different optimality criteria, the optimal form of the electrical dissipation operator $D[\psi]$. Once such an expression is determined the synthesis problem of a dissipative network governed by the so-found optimal form of equation (49) $)_{2}$ will be addressed.

The circuit depicted in Figure 10 is governed by a central finite difference approximation of the homogeneous version $(\beta=0)$ of equation $(49)_{2}$. In fact, the flux linkages denoted by $\varphi_{j}^{k}$, where $k$ indicates the "floor", are recognized to be determined by the following recursive relations

$$
\left\{\begin{array}{l}
\varphi_{j}^{1}=\frac{\psi_{j+1}-\psi_{j}}{n} \\
\varphi_{j}^{k}=\frac{\varphi_{j+1}^{k-1}-\varphi_{j}^{k-1}}{n}, \quad k=2, \ldots, M-1
\end{array},\right.
$$

in terms of the flux linkages $\psi_{j+1}$ and $\psi_{j}$.

On the other hand, the balance of currents at nodes $\varphi_{j}^{k}$ yields the following recursive set of relations for the currents flowing through the ports of the transformers connected to ground: 

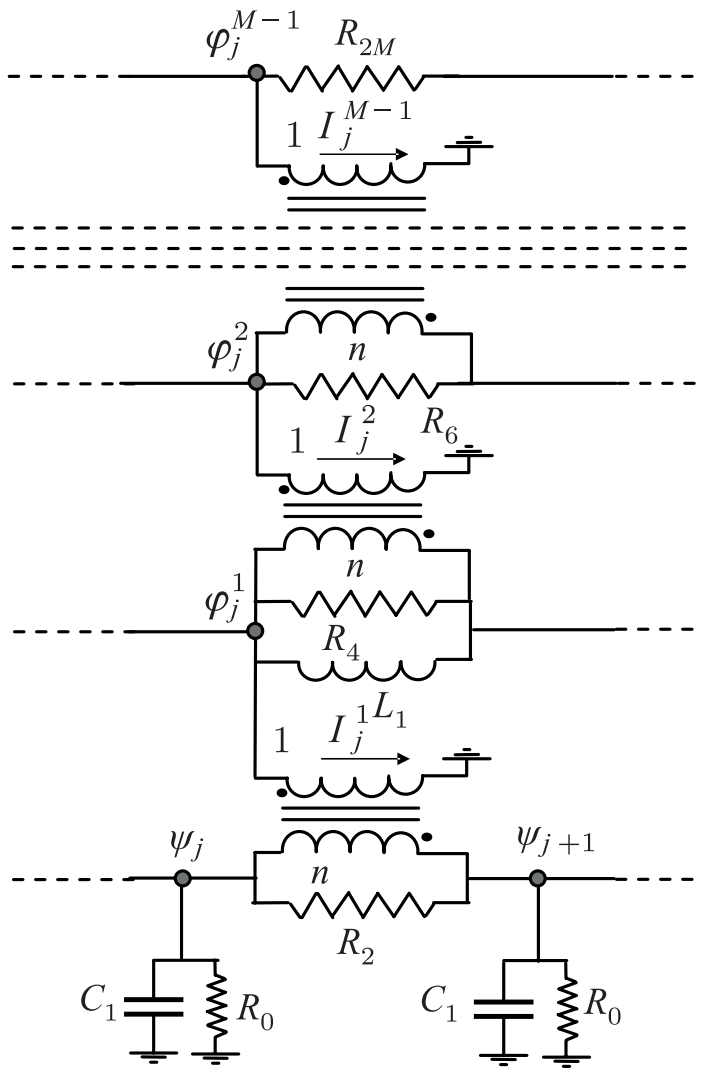

Figure 10. Electric analog circuit to the Euler beam, where dissipative elements are properly introduced to synthesize the generic operator $D[\cdot]$.

$$
\left\{\begin{array}{l}
I_{j}^{M-1}=\frac{1}{R_{2 M}}\left(\dot{\varphi}_{j+1}^{M-1}-2 \dot{\varphi}_{j}^{M-1}+\dot{\varphi}_{j-1}^{M-1}\right) \\
I_{j}^{k}=\frac{1}{R_{2 k+2}}\left(\dot{\varphi}_{j+1}^{k}-2 \dot{\varphi}_{j}^{k}+\dot{\varphi}_{j-1}^{k}\right)-\frac{1}{n}\left(I_{j}^{k+1}-I_{j-1}^{k+1}\right), \quad k=2, \ldots, M-2 \\
I_{j}^{1}=\frac{1}{R_{4}}\left(\dot{\varphi}_{j+1}^{2}-2 \dot{\varphi}_{j}^{2}+\dot{\varphi}_{j-1}^{2}\right)+\frac{1}{L_{1}}\left(\varphi_{j+1}^{2}-2 \varphi_{j}^{2}+\varphi_{j-1}^{2}\right)-\frac{1}{n}\left(I_{j}^{2}-I_{j-1}^{2}\right)
\end{array} .\right.
$$

The balance of currents at node $\psi_{j}$ yields in terms of the Lagrangian coordinates of the electrical lumped circuit:

$$
C_{1} \ddot{\psi}_{j}+\frac{1}{R_{0}} \dot{\psi}_{j}-\frac{1}{R_{2}}\left(\dot{\psi}_{j+1}-2 \dot{\psi}_{j}+\dot{\psi}_{j-1}\right)-\frac{1}{n}\left(I_{j}^{1}-I_{j-1}^{1}\right)=0 .
$$




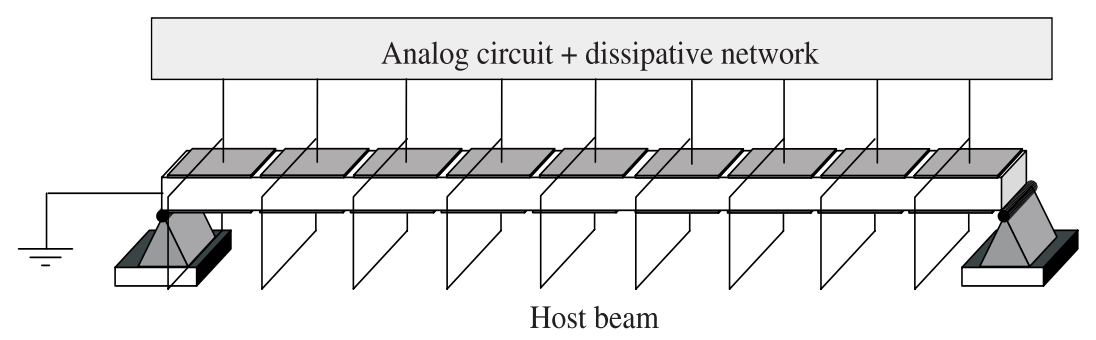

Figure 11. Simply-supported PEM beam.

From the recursive set of relations (51) it is possible to express the currents $I_{j}^{1}$ and $I_{j-1}^{1}$, appearing in equation (52), in terms of the set of flux linkages $\varphi_{j}^{k}$, which are themselves related to the independent coordinates $\psi_{j}$ by equation (50). Thus, it is easy to understand that, once the above-mentioned relations have been exploited, equation (52) represents a central finite difference approximation of the homogeneous version of equation $(49)_{2}$ in terms of the flux linkage field $\psi$, i.e.

$$
\begin{aligned}
& C_{1} \ddot{\psi}+\frac{(\Delta x)^{4}}{n^{2} L_{1}} \psi^{\mathrm{IV}}+\frac{1}{R_{0}} \dot{\psi}-\frac{(\Delta x)^{2}}{R_{2}} \dot{\psi}^{\prime \prime}+\frac{(\Delta x)^{4}}{n^{2} R_{4}} \dot{\psi}^{\mathrm{IV}}-\frac{(\Delta x)^{6}}{n^{4} R_{6}} \dot{\psi}^{\mathrm{VI}}+\ldots+ \\
+ & (-1)^{M+1} \frac{(\Delta x)^{2 M}}{n^{2 M-2} R_{2 M}} \dot{\psi}^{(2 M)}=0 .
\end{aligned}
$$

The grid step $\Delta x$ has been introduced in order to homogenize equation (52), according to the notation (7).

Non-dimensionalizing equation (53) it is easy to establish the following set of relations among the resistances of the resistors in the circuit depicted in Figure 10 and the real constants $y_{i}$ appearing in equation (48):

$$
\left\{\begin{array}{l}
y_{0}=-\varpi l^{4} \frac{n^{2} L_{1}}{(\Delta x)^{4} R_{0}} \\
y_{2 k}=(-1)^{k+1} \frac{\varpi L_{1}}{R_{2 k}}\left(\frac{n l}{\Delta x}\right)^{2(2-k)} \quad k=1, \ldots M
\end{array}\right.
$$

\section{APPLICATION SAMPLE: VIBRATIONS OF SIMPLYSUPPORTED DISSIPATIVE PEM BEAM}

In this section we address the problem of undamped and damped free vibrations of a simplysupported PEM beam (shown in Figure 11$)^{6}$ beam via a modal analysis (Section 4.1.). Subsequently (Sections 4.2. and 4.3.) we consider purely electrically dissipative PEM beams and determine the optimal dissipation operator in the specified class (48), the associated circuital topology and consequently the required optimal resistances. 
In order to achieve the aforementioned goals two different optimality criteria are adopted.

1. A criterion involving the mechanical strain energy time envelope (Juang, 1984), given by the shaded areas in Figure 15; such a criterion seems in the authors' opinion to be suitable when control of fatigue phenomena is needed (Section 4.2).

2. A "critical damping" criterion, often called the pole placement technique (see, for example, Hagood and von Flotow, 1991; Miller and Crawley, 1988), in which the time rate decay of free vibrations is maximized (Section 4.3).

\subsection{Modal Analysis}

In this subsection we perform a modal analysis for a simply-supported PEM beam ${ }^{7}$, based upon the Galerkin method.

The set of basis functions chosen to represent the deflection $u(x, t)$ and the flux linkage $\psi(x, t)$ of the PEM beam is constituted by the eigenfunctions $\varphi_{m}(x)$ of the spatial differential operator determining the modal forms for both the uncoupled motions:

$$
\varphi_{m}(x)=\sqrt{2} \sin (m \pi x), \quad m=1,2, \ldots
$$

Consequently, a Fourier expansion for the solution of equation (49) is considered

$$
\left[\begin{array}{l}
u(x, t) \\
\psi(x, t)
\end{array}\right]=\sum_{m=1}^{\infty}\left[\begin{array}{l}
p_{m}(t) \\
q_{m}(t)
\end{array}\right] \varphi_{m}(x)
$$

where $p_{m}(t)$ and $q_{m}(t)$ are respectively the mechanical and electrical Fourier coefficients of the expansion. With simple calculation the following system of ODEs governing the time evolution of a generic pair of electrical and mechanical Fourier coefficients is obtained, in which unitary dimensionless modal masses are made to appear:

$$
\begin{gathered}
{\left[\begin{array}{ll}
1 & 0 \\
0 & 1
\end{array}\right]\left[\begin{array}{c}
\ddot{p}_{m}(t) \\
\ddot{q}_{m}(t)
\end{array}\right]+\left[\begin{array}{cc}
\left(\frac{m \pi}{\alpha}\right)^{4} & 0 \\
0 & \left(\frac{m \pi}{\alpha}\right)^{4}
\end{array}\right]\left[\begin{array}{c}
p_{m}(t) \\
q_{m}(t)
\end{array}\right]+} \\
+\left[\begin{array}{cc}
0 & -\frac{\beta^{2}(m \pi)^{2}}{\alpha^{4}} \\
\frac{\beta^{2}(m \pi)^{2}}{\alpha^{4}} & \frac{P_{D}(m)}{\alpha^{4}}
\end{array}\right]\left[\begin{array}{c}
\dot{p}_{m}(t) \\
\dot{q}_{m}(t)
\end{array}\right]=\left[\begin{array}{l}
0 \\
0
\end{array}\right]
\end{gathered}
$$

where the polynomial $P_{D}(m)$ is defined by

$$
P_{D}(m)=\sum_{k=0}^{M}(-1)^{k+1} y_{2 k}(m \pi)^{2 k} .
$$

Let us explicitly remark that because of the performed choices, i.e. 
1. boundary conditions (simply-supported PEM beam);

2. basis functions (modal shapes of both the uncoupled electrical and mechanical systems);

3. class of dissipation operators (48);

we obtained a set of uncoupled evolution problems for each electromechanical mode. Thus, it is possible to easily derive the analytical solution for the Fourier coefficients; otherwise cumbersome numerical analysis should be performed.

For our purposes it will be more useful to consider the associated system in the state space form:

$$
\frac{\mathrm{d}}{\mathrm{d} t}\left[\begin{array}{c}
p_{m}(t) \\
q_{m}(t) \\
\dot{p}_{m}(t) \\
\dot{q}_{m}(t)
\end{array}\right]=\left[\begin{array}{cccc}
0 & 0 & 1 & 0 \\
0 & 0 & 0 & 1 \\
-a_{m} & 0 & 0 & c_{m} \\
0 & -a_{m} & -c_{m} & -d_{m}
\end{array}\right]\left[\begin{array}{c}
p_{m}(t) \\
q_{m}(t) \\
\dot{p}_{m}(t) \\
\dot{q}_{m}(t)
\end{array}\right]
$$

with:

$$
\left\{\begin{array}{rl}
a_{m} & =\frac{(m \pi)^{4}}{\alpha^{4}} \\
c_{m} & =\frac{\beta^{2}(m \pi)^{2}}{\alpha^{4}} \\
d_{m} & =\frac{P_{D}(m)}{\alpha^{4}}
\end{array}, \quad A_{m}=\left[\begin{array}{cccc}
0 & 0 & 1 & 0 \\
0 & 0 & 0 & 1 \\
-a_{m} & 0 & 0 & c_{m} \\
0 & -a_{m} & -c_{m} & -d_{m}
\end{array}\right] .\right.
$$

Let us remark that the dissipative parameter $d_{m}$ depends linearly on the set of dimensionless damping parameters $y_{i}$.

As a preliminary to the study of vibration damping, let us consider the conservative case, when $d_{m}=0$ (for more details on the analysis of non-dissipative system PEM beams, see Alessandroni et al., 2002). Indeed the solution of equation (59) shows a beating phenomenon in which the mechanical energy is transferred into the electrical form and vice versa. The dimensionless modal angular frequency of the "beating" envelope is

$$
\omega_{e}^{m}=\frac{2 \beta^{2}}{\alpha^{4} m^{2}},
$$

while the dimensionless carrier modal frequency is

$$
\omega_{c}^{m}=\sqrt{1+\left(\frac{\beta^{2}}{2 \alpha^{2}}\right)^{2}}\left(\frac{2}{m \alpha}\right)^{2} .
$$

Hence, a possible measure of the efficiency of the piezoelectric coupling can be chosen to be the beating ratio defined as the ratio of the envelope angular frequency and the carrier angular frequency:

$$
\frac{\omega_{e}^{m}}{\omega_{c}^{m}}=\frac{\beta^{2}}{2 \alpha^{2}} \frac{1}{\sqrt{1+\left(\beta^{2} / 2 \alpha^{2}\right)^{2}}}=\frac{k_{m e}}{2 \sqrt{\gamma K_{M}}} \frac{1}{\sqrt{1+\left(k_{m e} / 4 \gamma K_{M}\right)}} .
$$



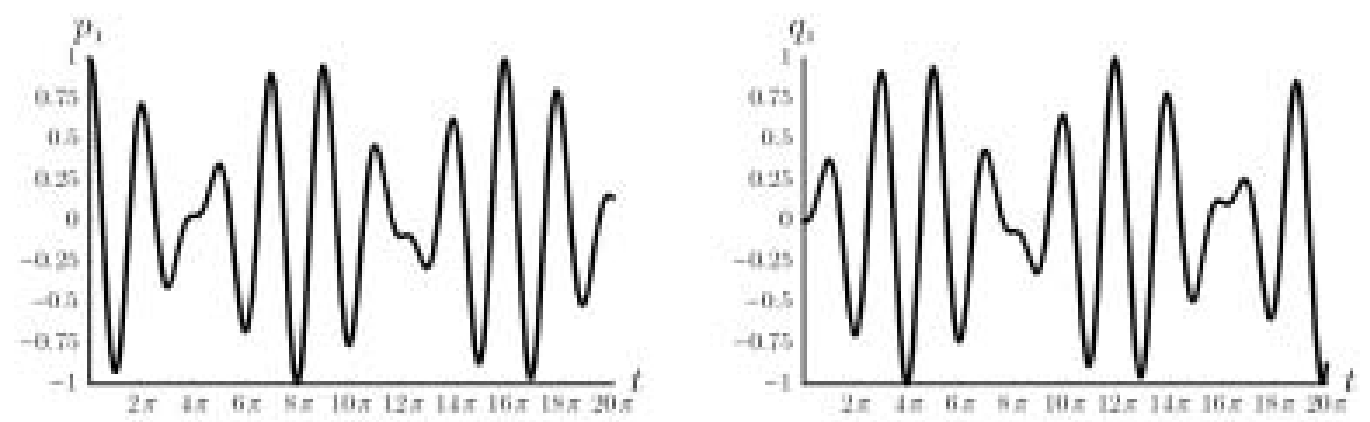

Figure 12. Time evolution of the first mechanical and electrical modes.

This coefficient is completely independent of the mode and for the aluminum beam introduced in Section 3.2.2. can be evaluated to be

$$
\frac{\omega_{e}^{m}}{\omega_{c}^{m}} \simeq 0.1225
$$

For illustrative purposes we consider for the introduced aluminum PEM beam the time evolution of the first mechanical and electrical modes for purely mechanical initial conditions of the form:

$$
\left\{\begin{array}{l}
p_{1}(0)=1 \\
q_{1}(0)=0 \\
\dot{p}_{1}(0)=0 \\
\dot{q}_{1}(0)=0
\end{array} ; \quad\left\{\begin{array}{l}
p_{m}(0)=0 \\
q_{m}(0)=0 \\
\dot{p}_{m}(0)=0 \\
\dot{q}_{m}(0)=0
\end{array}, \quad m>1\right.\right.
$$

We plot these in Figure 12.

\subsection{Resistance Optimizations by Infinite Horizon Minimization}

The objective of our optimization procedure is to minimize the strain energy time envelope of the vibrating beam for given initial conditions (for more details on this optimization technique, see Juang, 1984). Thus, let us define the following set of modal cost functions by which an optimal design of the dissipative elements can be pursued:

$$
J_{m}\left(y_{0}, \ldots y_{M}\right)=\frac{1}{2} \int_{0}^{\infty} a_{m} p_{m}^{2} \mathrm{~d} t=: \frac{1}{2} \int_{0}^{\infty}\left(z_{m}^{T} Q_{m} z_{m}\right) \mathrm{d} t .
$$

Here we have adopted the following matrix notation:

$$
z_{m}^{T}=\left[p_{m}, q_{m}, \dot{p}_{m}, \dot{q}_{m}\right], \quad Q_{m}=\left[\begin{array}{cccc}
a_{m} & 0 & 0 & 0 \\
0 & 0 & 0 & 0 \\
0 & 0 & 0 & 0 \\
0 & 0 & 0 & 0
\end{array}\right] .
$$


By taking the limit as time goes to infinity (infinite horizon minimization), we are seeking a time-independent solution of the optimization problem. Let us explicitly remark that $\frac{1}{2} z_{m}^{T}(t) Q_{m} z_{m}(t)$ represents the generic mechanical strain modal energy at time $t$.

We can now show that there exists a value of $M$ and a consequent choice of the damping coefficients $y_{1}, \ldots y_{M}$ which allows for a simultaneous minimization of every modal cost function for every initial set of mechanical modal displacements $\left\{p_{1}^{0}, p_{2}^{0}, \ldots\right\}$.

Indeed, as the system is dissipative (i.e. the real part of the eigenvalues of the state matrix $A_{m}$ in equation (59) are negative) for every choice of $y_{i}$ the modal cost function can be written as

$$
J_{m}\left(y_{0}, \ldots y_{M}\right)=-\frac{1}{2}\left(z_{m}^{0}\right)^{T} P_{m} z_{m}^{0},
$$

where $z_{m}^{0}$ is the modal initial condition to be considered. $P_{m}$ is the unique solution of the following Lyapunov equation

$$
A_{m}^{T} P_{m}+P_{m} A_{m}=Q_{m},
$$

where $A_{m}$ is the state matrix in equation (59).

Equation (69) yields

$$
P_{m}\left(y_{0}, \ldots y_{M}\right)=\left[\begin{array}{cccc}
-\frac{\left(a_{m}+c_{m}^{2}\right)}{2 d_{m}}-\frac{a_{m} d_{m}}{2 c_{m}^{2}} & -\frac{a_{m}}{2 c_{m}} & -\frac{1}{2} & -\frac{c_{m}}{2 d_{m}} \\
-\frac{a_{m}}{2 c_{m}} & -\frac{\left(a_{m}+c_{m}^{2}\right)}{2 d_{m}} & \frac{c_{m}}{2 d_{m}} & 0 \\
-\frac{1}{2} & \frac{c_{m}}{2 d_{m}} & -\frac{1}{2 d_{m}}-\frac{d_{m}}{2 c_{m}^{2}} & \frac{1}{2 c_{m}} \\
-\frac{c_{m}}{2 d_{m}} & 0 & \frac{1}{2 c_{m}} & -\frac{1}{2 d_{m}}
\end{array}\right]
$$

Considering only modal mechanical initial displacement, the generic modal cost function becomes

$$
J_{m}=-\frac{1}{2}\left(p_{m}^{0}\right)^{2} P_{m_{11}} .
$$

Substituting the aforementioned expression for the matrix $P_{m}$ we finally obtain

$$
J_{m}=\frac{1}{2}\left(p_{m}^{0}\right)^{2}\left(\frac{\left(a_{m}+c_{m}^{2}\right)}{2 d_{m}}+\frac{a_{m} d_{m}}{2 c_{m}^{2}}\right) .
$$

Differentiating this expression with respect to the dimensionless damping parameters $y_{i}$ and equating to zero we find the following $M+1$ conditions:

$$
\frac{\partial J_{m}}{\partial y_{k}}=\frac{\mathrm{d} J_{m}}{\mathrm{~d} d_{m}} \frac{(-1)^{k+1}(m \pi)^{2 k}}{2 \alpha^{4}}=0, \quad k=0, \ldots M .
$$

These are simultaneously satisfied if and only if 


$$
\frac{\mathrm{d} J_{m}}{\mathrm{~d} d_{m}}\left(y_{0}, \ldots y_{M}\right)=0
$$

which yields

$$
d_{m}^{o p t}=c_{m} \sqrt{1+\frac{c_{m}^{2}}{a_{m}}} .
$$

The minimum value attained by the modal cost function is

$$
J_{m_{\min }}=\frac{1}{2}\left(p_{m}^{0}\right)^{2} \sqrt{\left(\frac{a_{m}}{c_{m}^{2}}+1\right)} \sqrt{a_{m}},
$$

which is clearly independent of the value of the dimensionless damping parameters $y_{i}$, but depends exclusively on the electromechanical parameters $\alpha$ and $\beta$ of the non-dissipative PEM beam. In particular, the minimum value of the modal cost function can be easily written as follows

$$
J_{m_{\min }}=\bar{U}_{m} \frac{T_{m}}{\pi} \sqrt{1+\frac{\gamma K_{M}}{k_{m e}}},
$$

where $T_{m}$ is the dimensionless period of free vibrations for the $m$ th mode $\left(T_{m}=2 \pi / \sqrt{a_{m}}\right)$ and $\bar{U}_{m}$ is the dimensionless mean value of the mechanical modal strain energy for free vibrations, i.e.

$$
\bar{U}_{m}=\frac{1}{T_{m}} \int_{0}^{T_{m}} \frac{1}{2} a_{m}\left(p_{m}^{0} \cos \left(\frac{2 \pi}{T_{m}} t\right)\right)^{2} \mathrm{~d} t=\frac{1}{4} a_{m}\left(p_{m}^{0}\right)^{2} .
$$

Now let us determine the values of the damping parameters in the analog circuit substituting the expressions in equation (60) into equation (75):

$$
\frac{P_{D}(m)}{\alpha^{4}}=(m \pi)^{2} \frac{\beta^{2}}{\alpha^{4}} \sqrt{1+\left(\frac{\beta}{\alpha}\right)^{4}} .
$$

This last equation characterizes a surface of damping parameters all points of which optimize the cost function $J_{m}$. However, according to equation (58), the following choice

$$
\left\{\begin{array}{l}
y_{2 k}^{o p t}=0, \quad k=0,2,3 \ldots M \\
y_{2}^{o p t}=\beta^{2} \sqrt{1+\left(\frac{\beta}{\alpha}\right)^{4}}
\end{array}\right.
$$

allows for the simultaneous minimization of the cost functions $J_{m}$ for all $m$.

It is suggested to interpret the previous algebraic results in a geometrical way. In the space of "damping parameters" $\left\{y_{k}\right\}$, the optimal values for the $m$ th mode belongs to the hypersurface characterized by equation (74). Formula (80) shows that there exists a unique choice of damping parameters belonging to all the modal optimal hypersurfaces. 


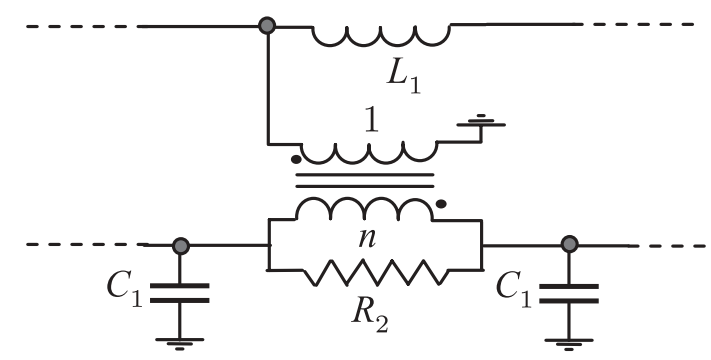

Figure 13. Dissipative circuit satisfying the optimality condition.

Hence the optimal electrical dissipative differential operator efficient for simultaneously for all modes is

$$
D[\dot{\psi}]=y_{2}^{o p t} \dot{\psi} \psi^{\prime \prime} .
$$

Once the optimal dissipation operator has been established, a suitable dissipative electrical network has to be connected to the analog electrical circuit in Figure 5 in order to obtain the following homogenized evolution equation:

$$
\psi^{\mathrm{IV}}+\alpha^{4} \ddot{\psi}-\beta^{2} \dot{u}^{\prime \prime}-y_{2}^{o p t} \dot{\psi}^{\prime \prime}=0 .
$$

This dissipative network is easily synthesized from Figure 11 interconnecting the capacitors by a set of resistors as in Figure 13. The value of the resistances can be obtained from equation (54):

$$
R_{2}=\frac{L_{1} n^{2}}{y_{2}(\Delta x)^{2}} \varpi l^{2} .
$$

In order to verify the optimality condition (80) the value of the resistances has to be chosen as follows

$$
R_{2}^{\text {opt }}=\frac{L_{1} n^{2}}{y_{2}^{\text {opt }}(\Delta x)^{2}} \varpi l^{2}=\frac{L_{1} n^{2} \varpi l^{2}}{(\Delta x)^{2} \beta^{2} \sqrt{1+(\beta / \alpha)^{4}}}=\sqrt{\frac{\rho}{\gamma}} \frac{(\Delta x)}{k_{m e} \sqrt{1+\left(k_{m e} / K_{M}\right)(1 / \gamma)}}
$$

For the aluminum PEM beam introduced in Section 3.2.2. we obtain the following value for the damping parameter $y_{2}$

$$
y_{2}^{o p t} \simeq 2.509
$$

and as a consequence the resistance is determined by

$$
R_{2}^{o p t} \simeq 4.690 \mathrm{k} \Omega .
$$




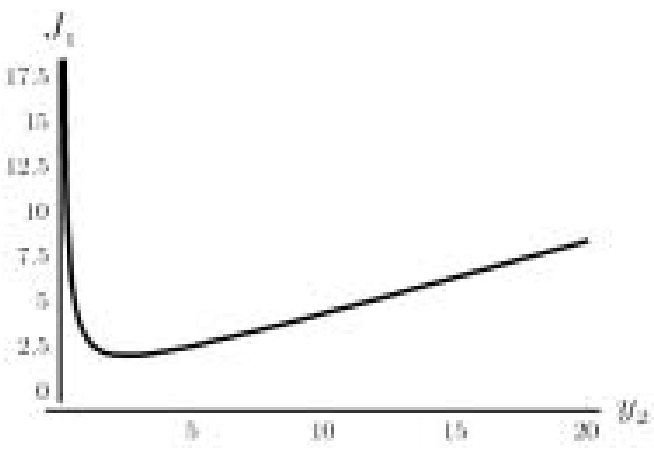

Figure 14. First modal cost function.

In Figure 14 we plot the graphics of the cost function for the first mode as a function of the parameter $y_{2}$, assuming unitary initial condition, where the minimum value achieved is given by

$$
J_{1_{\min }} \simeq 2.086 .
$$

Let us exhibit in Figure 14 the evolution of the first modal strain energies for three different values of the damping parameter; the shaded area is the value of introduced cost function $J_{1}$.

\subsection{Resistance Optimization by Pole Placement Technique}

Another possibility for choosing the coefficient $y_{i}$ in the dissipation operator (48) to be employed in the electrical analog is provided by a different optimality criterion, i.e. the pole placement technique widely discussed in Hagood and von Flotow (1991) and Miller and Crawley (1988). The pole placement criterion is based on a cost function of a different form with respect to equation (66). Once the $m$ th modal time rate decay is defined by

$$
\tau_{m}\left(y_{0}, \ldots y_{M}\right)=\min _{i=1, \ldots, 4}\left[\left|\operatorname{Re}\left[\lambda_{i}^{m}\right]\right|\right],
$$

where $\lambda_{i}^{m}$ are the eigenvalues of the state space matrix $A_{m}$, it is necessary that the chosen values $\left\{y_{i}\right\}$ must maximize $\tau_{m}$.

Considering the eigenvalues of the generic state matrix $A_{m}$ it is easy to show that $\tau_{m}$ is maximized when

$$
d_{m}=2 c_{m},
$$

and in this case there are only two complex conjugate eigenvalues of multiplicity two (see, for example, Miller and Crawley, 1988). For this last circumstance, the present optimization 


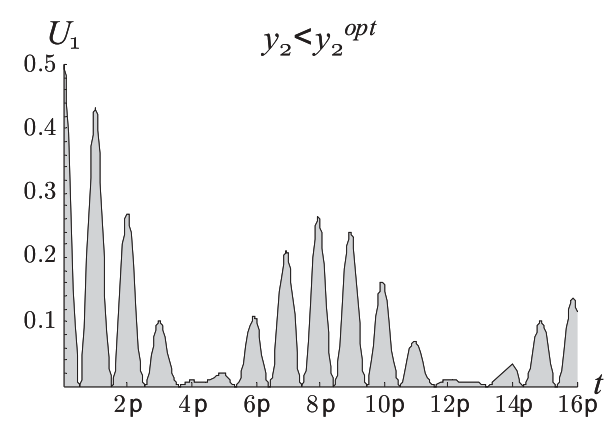

a)

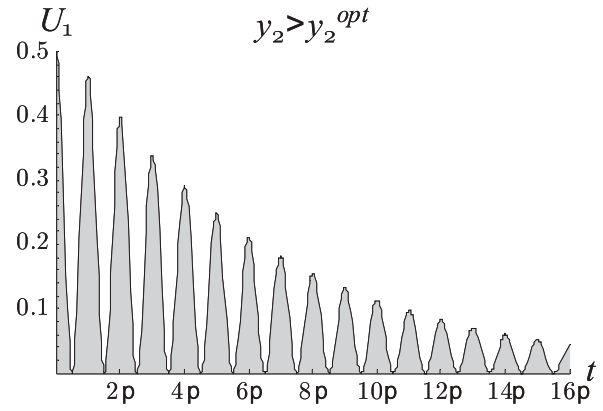

b)

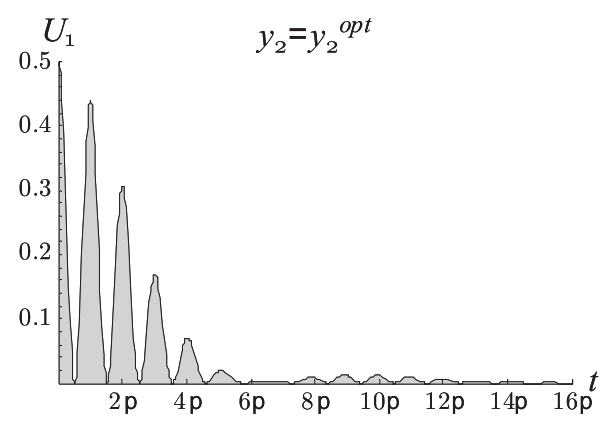

c)

Figure 15. Time evolution of the first modal strain energy for (a) resistance larger than the optimal value, (b) resistance smaller than the optimal value, and (c) optimal resistance.

criterion is often called the critical damping criterion. The criticalness of the abovementioned criterion is to be ascribed to the time vibration envelope which switches (according with increasing values of bifurcation parameters $d_{m}$ ) from underdamped to overdamped decay. The maximum time rate decay $\tau_{m}^{\text {opt }}$ can be easily evaluated to be equal to $c_{m} / 2$.

The imaginary parts of the eigenvalues $\lambda_{i}^{m}$ evaluated at $d_{m}=2 c_{m}$ are recognized to be equal to $\pm \sqrt{a_{m}-\left(c_{m} / 2\right)^{2}}$. Hence, the damping ratio evaluated when condition (89) holds becomes

$$
\zeta_{m}=\frac{c_{m} / 2}{\sqrt{\left(c_{m} / 2\right)^{2}+\left(\sqrt{a_{m}-\left(c_{m} / 2\right)^{2}}\right)^{2}}}=\frac{c_{m}}{2 \sqrt{a_{m}}} .
$$

Taking into account relations (60) it is easy to see that

$$
\zeta_{m}=\frac{\beta^{2}}{2 \alpha^{2}}=\frac{k_{m e}}{2} \sqrt{\frac{1}{K_{M} \gamma}}:=\zeta
$$


hence, the damping ratio of the $m$ th mode, once condition (89) has been satisfied, does not depend on the mode number $m$.

It is still possible to accomplish this optimality condition for each mode by means of a unique choice of the resistances. In fact, choosing $d_{m}=2 c_{m}$ we obtain

$$
\frac{P_{D}(m)}{\alpha^{4}}=2(m \pi)^{2} \frac{\beta^{2}}{\alpha^{4}},
$$

which can be satisfied, according to equation (58), for every mode by choosing

$$
\left\{\begin{array}{l}
y_{k}^{o p t}=0, \quad k=0,1,3 \ldots M \\
y_{2}^{o p t}=2 \beta^{2}
\end{array}\right.
$$

Once again, the interconnection to be used is simply constituted by a set of resistors interconnecting the PZT capacitors, and the value of these resistors is determined by

$$
R_{2}^{o p t}=\frac{L_{1} n^{2}}{y_{2}^{o p t}(\Delta x)^{2}} \varpi l^{2}=\frac{1}{2} \frac{(\Delta x)}{k_{m e}} \sqrt{\frac{\rho}{\gamma}} .
$$

For the PEM beam designed in the previous section the pole placement optimization criterion yields

$$
y_{2}^{o p t} \simeq 4.873
$$

and consequently the optimal resistance is found to be

$$
R_{2}^{o p t} \simeq 2.415 \mathrm{k} \Omega
$$

In Figure 16 we show the detail of the first mode root locus in the neighborhood of the bifurcation (critical) point, which corresponds to the optimal value for the resistance, as the dissipative parameter $d_{1}$ varies from 0 to infinity.

The optimal damping ratio achieved by the pole placement criterion is equal to

$$
\zeta \simeq 12.34 \%
$$

In order to show that the particular connection for the resistances in the analog circuit realized by $R_{2}$ allows for the simultaneous optimal damping for each mode, let us show in Figure 17 the root locus for the second and third modes. Condition (91) establishes that the damping ratio is a parameter independent of the specific $m$ th mode, and thus all the critical points (occurring at the same resistance $R_{2}$ ) in the complex plane have the same phase, while the modules are found to be equal to $a_{m}$.

The crosses in the root loci depicted in Figures 16 and 17 represent the roots determined for vanishing $y_{2}$, while the empty circles indicate the roots for infinite value of $y_{2}$ and the arrows denote the versus of the $y_{2}$ increment. 


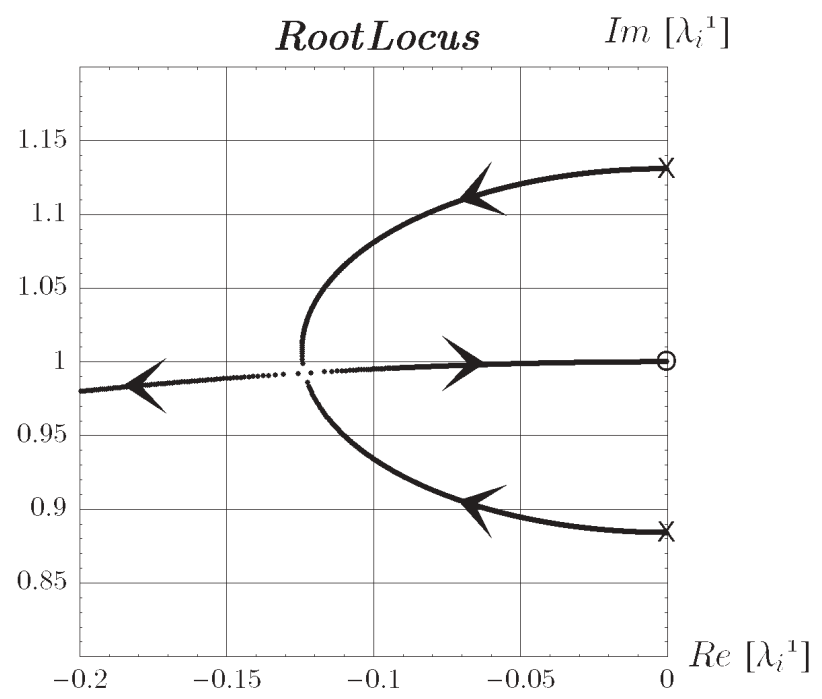

Figure 16. First mode root locus for increasing value of the damping parameter $y_{2}$.

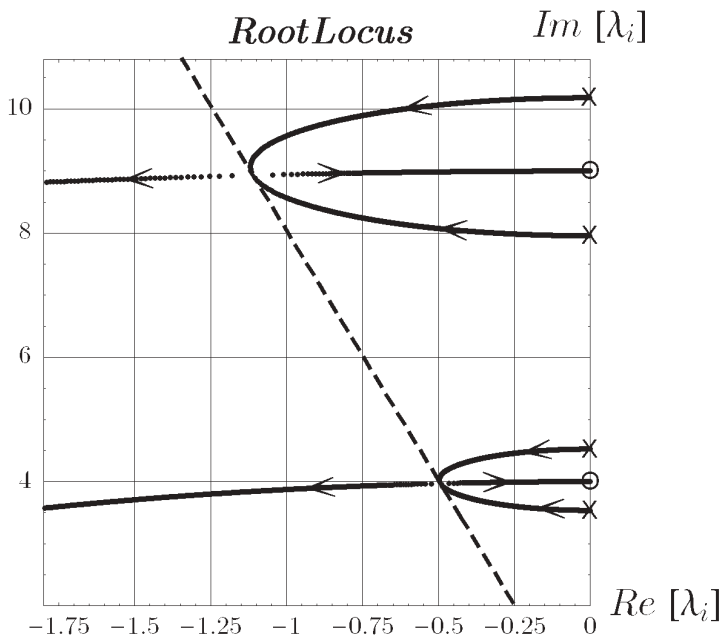

Figure 17. Root locus for the second and third modes, increasing the value of $y_{2}$.

\section{CONCLUSIONS}

A novel completely passive device for multimodal structural vibration damping based on distributed piezoelectric effect has been conceived and designed for engineering applications. The novel passive smart structure consists of a host beam, an array of bender transducers uniformly distributed on it, and an analog passive circuit completed by dissipative elements 
interconnecting the terminals of the piezo-transducers. Such an analog passive circuit can be regarded as a distributed electric controller for mechanical vibrations. The introduced PEM beam allows for an efficient multimodal vibration damping and control in despite of inductances of extremely reduced value in the analog circuit and low-cost piezoelectric transducers. It has to be stressed that, to our knowledge, in the literature the problem of structural mechanical vibration suppression is never addressed by combining the two concepts of

1. distributed piezoelectric actuation and

2. multiresonant passive electric control.

The device considered in the present paper ensures a multifrequency and modalform-adapted energy transduction, and consequently the simultaneous multimodal vibration suppression. Indeed, we may state that the electric controller can respond to mechanical excitation with exactly the same electrical modal forms and frequencies as the mechanical structure, and that the piezoelectric transducers are consequently bound to exert forces in a synergetic way in the absence of any external control law. In a suggestive way, the electric controller interconnecting the piezoelectric transducers is synthesized for resonating at the same frequencies as the structural element to be damped. However, in addition, it is designed to respond by driving every transducer with the electric potential needed to suppress every mechanical modal forms eventually excited.

Finally we fixed two optimality dissipation criteria and managed to find a placement (in the electric circuit topology) of resistors which ensured the simultaneous optimal damping of every mechanical excitation. This result is not always possible, as is well known by the theory of control, and is related to the use of the electromechanical analogy which we have exploited. The two optimality criteria refer to different mechanical phenomena: the first is related to the strain energy stored in the structure during the whole course of vibration, the second is associated with the time rate decay of vibration. However, they provide:

1. technological feasibility of the entire passive control device;

2. the same placement for the resistors in the circuit;

3. comparable values for the optimal resistances.

As far as point 3 is concerned, and as can be reasonably expected, the two used criteria yield slightly different, yet not coincident, resistances. Such difference can be explained on the basis of the following comment: the former criterion refers to a purely mechanical feature (strain energy stored during the whole course of vibration), while the latter, which is widely adopted in the literature, does not allow us to distinguish electric and mechanical contributions in the overall energy.

The main limit of the effectiveness of the presented controller is due to the lumped nature of the employed electric circuit and piezoelectric transduction devices. This circumstance obviously approximately imposes as a lower bound to the wavelength of mechanical modes which may be damped the size of the typical piezoelectric actuator used.

The design of an experiment to validate the novel concepts and methodology is addressed for an aluminum beam, considering available PZT transducers and practically interesting results are exhibited via analytical and numerical techniques. With a view towards the construction of a demonstrative prototype it has to be remarked that: 
1. the topology of the analog circuit is extremely simple;

2. the analog circuit can be entirely realized by means of light, low-cost, passive elements, dispensing the use of any external feeding;

3. an efficient multimodal control of mechanical vibrations is guaranteed;

4. mechanical vibrations are rapidly suppressed by means of suitable resistors properly inserted in the analog circuit.

Future theoretical and experimental investigations should include:

1. the study of more complex PEM structures constituted by different structural elements, as beams, plates and shells;

2. the study of PEM trusses as fundamental structural elements in aerospace technology;

3. the development of a finite element code for the analysis of PEM structures taking into account the finite degrees of freedom of the electrical analog circuit and the comparison with the results achieved by the homogenized distributed model;

4. the optimization of dissipative circuits for specific tasks as in forced vibrations of PEM structures.

As far as point 1 is concerned, special interest will be addressed to the problem of multimodal vibration control of plates; in more detail, we will face the synthesis of a lumped circuit analog to a Kirchhoff-Love plate discretized via finite difference method. As already performed for beams, in the present paper, our effort will be focused on synthesizing an analog circuit which can dispense with the use of any external feeding. Coupling of a mechanical plate and electric network will be worked out exploiting a uniformly distributed array of piezoelectric transducers. The homogenization of the PEM plate will be achieved exploiting an energy identification method. The vibration suppression will be ensured by resistors properly inserted in the analog circuit.

Acknowledgments. The partial support of the Engineering Science and Mechanics Department of Virginia Polytechnic Institute and State University is gratefully acknowledged by the authors. This presented research has been also partially supported by MIUR, Ministero per l'Innovazione, l'Università e la Ricerca, Fondi Ricerca PRIN "Sintesi di circuiti piezoelettrici e tecniche di disaccoppiamento per il controllo di vibrazioni meccaniche" (protocollo 200109788_003) and by Ricerche di Ateneo (Università di Roma "La Sapienza") "Controllo attivo e passivo delle vibrazioni strutturali mediante modelli continui e discreti" (codice progetto: C26A013522). The authors wish to thank warmly Ing. Pier Mario Pollina, who read the manuscript with fresh eyes and helped to correct imperfections.

\section{NOTES}

1. Nevertheless, the problem may be solved also in a different way, looking for an analog circuit where the displacements are represented by stored charges and the contact actions by voltage drops.

2. The piezo-layer constituted by distinct patches is assumed to completely cover the whole beam span.

3. Once the characteristic frequency has been chosen equal to $\varpi$, then the scaling parameter for the time variable is assumed to be $1 / \varpi$.

4. This condition establishes that the characteristic capacitive energy in the analog circuit has to be equal to the characteristic kinetic energy of the vibrating beam. 
5. The sign of each constant $y_{2 k}$ has to be established in order to guarantee the passivity of the electrical circuit.

6. The electrical simply-supporting conditions establish that the bender transducer at the beam left edge has to be short-circuited to ground. Thus it can be omitted, since it does not contribute to the energy transfer between the electrical and the mechanica system.

7. Homogeneous electrical boundary conditions of the form $\psi^{2 j}(0, t)=\psi^{2 j}(1, t)=0$ with $j$ varying from 0 to $\max \{2(M-1), 1\}$ are considered.

\section{REFERENCES}

Alessandroni, S., dell'Isola, F., and Porfiri, M., 2002, “A revival of electric analogs for vibrating mechanical systems aimed to their efficient control by PZT actuators," International Journal of Solids and Structures 39(20), 52955324.

Crandall, S. H., Karnopp, D. C., Kurtz, E. F. Jr., and Pridmore-Brown, F. C., 1968, Dynamics of Mechanical and Electromechanical Systems, McGraw-Hill, New York.

Crawley, E. F., 1994, "Intelligent structures for aerospace: A technology overview and assessment," AIAA Journal 32(8), 1689-1699.

Crawley, E. F. and de Luis, J., 1987, "Use of piezoelectric actuators as elements of intelligent structures," AIAA Journal 25(10), 1373-1385.

dell'Isola, F. and Vidoli, S., 1998, "Continuum modelling of piezoelectromechanical truss beams: An application to vibration damping," Archive of Applied Mechanics 68, 1-19.

Forward, R. L., 1981, "Electronic damping of orthogonal bending modes in a cylindrical mast - experiment," Journal of Spacecraft and Rockets 18,11-17.

Forward, R. L. and Swigert, G. J., 1981, "Electronic damping of orthogonal bending modes in a cylindrical mast theory," Journal of Spacecraft and Rockets 18, 5-10.

Gantmacher, F. R., 1980, Lezioni di Meccanica Analitica, Edizioni Mir, Mosca (in Italian).

Hagood, N. W. and von Flotow, A., 1991, "Damping of structural vibrations with piezoelectric materials and passive electrical networks," Journal of Sound and Vibrations 146, 243-268.

Hanagud, S., Obal, M. W., and Meyyappa, M., 1985, "Electronic damping techniques and active vibration control," in Proceedings of the 26th AIAA/ASME/ASCE/AHS 26th Structures, Structural Dynamics and Materials Conference, Orlando, FL, April, AIAA Paper 85-0752.

Hanagud, S., Obal, M. W., and Calise, A. J., 1992, "Optimal vibration control by the use of piezoceramic sensors and actuators," Journal of Guidance, Control, and Dynamics 15(5), 1199-1206.

Hollkamp, J. J., 1994, "Multimodal passive vibration suppression with piezoelectric materials and resonant shunts," Journal of Intelligent Material Systems and Structures 5, 49-57.

Institute of Electrical and Electronic Engineers (IEEE), 1987, "IEEE Standard on Piezoelectricity," Std 176-1987.

Juang, J. N., 1984, "Optimal design of a passive vibration absorber for a truss beam," Journal of Guidance, Control, and Dynamics 7(5), 733-739.

Kader, M., Lenczner, M., and Mrcarica, Z., 2001, "Distributed control based on distributed electronic circuits: application to vibration control," Microelectronics Reliability 41, 1857-1866.

Karplus, W. J. and Soroka, W. W., 1959, Analog Methods: Computation and Simulation, McGraw-Hill, New York.

Mason, W. P., 1950, Piezoelectric Crystals and their Applications to Ultrasonics, Van Nostrand-Reinhold, Princeton, NJ.

Meirovitch, L., 1974, “A new method of solution of the eigenvalue problem for gyroscopic systems," AIAA Journal 12, 1337-1342.

Meirovitch, L., 1975, “A modal analysis for the response of linear gyroscopic systems," Journal of Applied Mechanics 42, 446-450.

Meirovitch, L., 2000, Fundamentals of Vibrations, McGraw-Hill, Boston, MA.

Miller, D. W. and Crawley, E. F., 1988, "Theoretical and experimental investigation of space-realizable inertial actuation for passive and active structural control," Journal of Guidance, Control, and Dynamics 11(5), 449-458.

Niezreski, C., Brei, D., Balakrishnam, S., and Moskalik, A., 2001, "Piezoelectric actuation: State of the art," The Shock and Vibration Digest 33(4), 269-280. 
Richards, T. H., 1977, Energy Methods in Stress Analysis: With an Introduction to Finite Element Techniques, Halsted Press, New York.

Soedel, W., 1993, Vibrations of Shells and Plates, 2nd edition, Marcel Dekker, NY.

Vidoli, S. and dell'Isola, F., 2000, "Modal coupling in one-dimensional electromechanical structured continua," Acta Mechanica 141, 37-50.

Yang, B., 1991, "Eigenvalue inclusion principles for distributed gyroscopic systems," Vibration Analysis - Analytical and Computational, ASME 37, 7-12. 